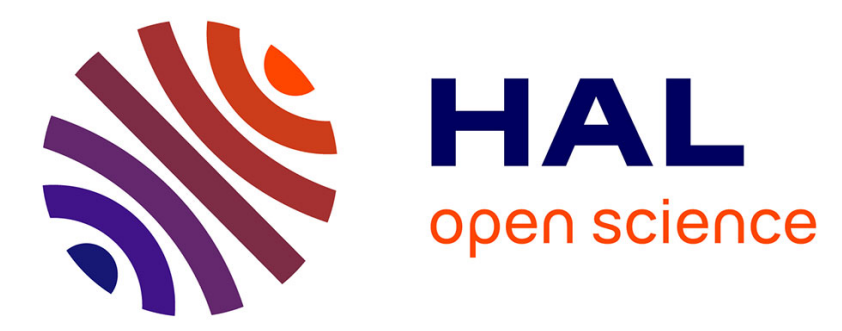

\title{
A class of fast-slow models for adaptive resistance evolution
}

Pastor E. Pérez-Estigarribia, Pierre-Alexandre Bliman, Christian E Schaerer

\section{To cite this version:}

Pastor E. Pérez-Estigarribia, Pierre-Alexandre Bliman, Christian E Schaerer. A class of fast-slow models for adaptive resistance evolution. Theoretical Population Biology, 2020, 135, pp.32-48. 10.1016/j.tpb.2020.07.003 . hal-02418511v2

\section{HAL Id: hal-02418511 https://hal.inria.fr/hal-02418511v2}

Submitted on 18 Dec 2020

HAL is a multi-disciplinary open access archive for the deposit and dissemination of scientific research documents, whether they are published or not. The documents may come from teaching and research institutions in France or abroad, or from public or private research centers.
L'archive ouverte pluridisciplinaire HAL, est destinée au dépôt et à la diffusion de documents scientifiques de niveau recherche, publiés ou non, émanant des établissements d'enseignement et de recherche français ou étrangers, des laboratoires publics ou privés. 


\title{
A class of fast-slow models for adaptive resistance evolution
}

\author{
Pastor E. Pérez-Estigarribia* ${ }^{*}$ Pierre-Alexandre Bliman ${ }^{\dagger} \quad$ Christian E. Schaerer $^{\ddagger}$
}

December 18, 2020

\begin{abstract}
Resistance to insecticide is considered nowadays one of the major threats to insect control, as its occurrence reduces drastically the efficiency of chemical control campaigns, and may also perturb the application of other control methods, like biological and genetic control. In order to account for the emergence and spread of such phenomenon as an effect of exposition to larvicide and/or adulticide, we develop in this paper a general time-continuous population model with two life phases, subsequently simplified through slow manifold theory. The derived models present density-dependent recruitment and mortality rates in a non-conventional way. We show that in absence of selection, they evolve in compliance with Hardy-Weinberg law; while in presence of selection and in the dominant or codominant cases, convergence to the fittest genotype occurs. The proposed mathematical models should allow for the study of several issues of importance related to the use of insecticides and other adaptive phenomena.
\end{abstract}

Keywords: Adaptive evolution, Insecticide resistance, Slow manifold theory, Stability, HardyWeinberg Law.

\section{Introduction}

\subsection{Insect pests and insecticide use}

A tiny insect is one of the deadliest animals for human: the mosquitoes. Nearly 700 million people contract diseases transmitted by mosquito every year [1]. Just for the transmission of malaria, almost a million people die every year [2], and 3.4 billion people are at risk worldwide [3]. Amongst the 150 arboviruses that cause diseases in humans, about 20 that are transmitted by mosquitoes are of primary medical importance [4. In particular dengue, transmitted by the primary vector Aedes aegypti and whose human and economic costs are staggering, is ranked as the most important mosquito-borne viral disease with epidemic potential in the world 5, 6. On the whole, vector-borne diseases generate a heavy public health problem and high economic cost in many world regions.

Besides, it has been suggested that insects destroy about $20 \%$ of the annual production of crops worldwide [7. The agricultural damage caused by insects may be direct, as well as indirect, through the transmission of plant diseases 8]. Two factors contribute to the importance of insects as agricultural pests: their diversity - two thirds of known species, around 600,000, are phytophagous - , and the fact that practically all plant species are consumed by at least one species of phytophagous insect- even some agricultural pests can hit many species of plants 9 . The issues induced by agricultural pests are therefore a challenge for global food production.

The most commonly used methods to suppress or reduce insect populations are based on chemical treatment. Conventional control strategy of mosquito populations use larvicides and/or adulticides [10. However, this strategy is affected by the evolution of resistance [11] which reduces the efficiency of the

*Polytechnic School, National University of Asunción, P.O. Box 2111 SL, San Lorenzo, Paraguay, email: pestigarribia@est.pol.una.py

†Sorbonne Université, Université Paris-Diderot SPC, Inria, CNRS, Laboratoire Jacques-Louis Lions, équipe Mamba, 75005 Paris, France. Corresponding author, email: pierre-alexandre.bliman@inria.fr

${ }^{\ddagger}$ Polytechnic School, National University of Asunción, P.O. Box 2111 SL, San Lorenzo, Paraguay, email: cschaer@pol.una.py 
chemical control campaigns and their lifespan [12. Nowadays, in addition to an alarming propagation of vectors, most of the species involved are showing resistance to many kinds of insecticides [13. Resistance not only reduces the efficiency of chemical control methods, but may also perturb the application of other control methods, like biological and genetic controls, through the undesired fitness advantage that it provides to the local mosquito species [14].

\subsection{Modelling of insecticide resistance evolution}

Resistance to insecticides is a man-made example of selection 15. This occurs when the environment is changed artificially by impregnation with an insecticide that increases the mortality of a target species, in such a way that some genetic variants survive better than others.

The issue of insecticide resistance provides a contemporary natural model to study how new adaptations evolve by selection: the selection agent is known - as an example a given insecticide-, the evolution is recent and rapid - few years after the application-, and the biological and genetic mechanisms are often known - many insect genes that code the targets for insecticides have been identified and cloned [16. In this regard, due to the cost, risk and logistical difficulties in the field study of resistance evolution, mathematical models may help to improve management strategies of insecticides, apart from allowing to learn more about adaptive evolution in the context of complex life history.

Literature related to models of evolution by selection is quite extensive. For an overview of the state of knowledge, the reader is referred to the following classical texts [17, 18, 19, 20, 21. Reproduction and natural selection involve inherently stochastic effects, and a typical modeling approach includes stochastic time-discrete processes 22, which may however present considerable mathematical complexity 21. In classical population genetics selection models, it is common to appeal to the law of large numbers and assume an infinite population size, to omit the effect of stochasticity and to focus the mathematical analysis on the evolution of the allelic and genotypic relative frequencies by selection. This is the case of Fisher selection equation (see 23, 24, 17), generally framed in evolutionary game theory as a replicator equation, to describe the allelic or genotype evolution of relative frequencies [19, 23. In this context, 17. provides models and analytical results of selection for some game dynamics equations, including continuous selection dynamics models such as: independent and density-dependent Malthusian fitness, additive and multiplicative fertility, and Mendelian populations.

For simplicity, it is common in population genetics, that the elementary mechanisms by which fitness is reduced are not specified [25, 26. On the other hand, the use of larvicides and/or adulticides for the control of mosquitoes affects the viability differently in the pre-reproductive and reproductive phases. In addition, viability may decrease in a growing population due to the limitation of some resources by competition, and may depend on density, which in turn may affect different classes of individuals in the population in a non-homogeneous way.

Several approaches have been proposed in the literature to model insecticide resistance, with several objectives. In [27] a time-discrete model is presented for three genotypes that considers a resistance allele with intermediate dominance in an autosomal locus. A time-discrete model of insecticide resistance is developed in 28 that includes migration, in order to understand variations in the time required for insects to develop resistance. Likewise, [29] explored a model proposed in [30] to quantify changes in gene frequency between zygotes in each generation. The model in question is time-discrete, with intermediate dominance alleles in an autosomal locus, and assumes migration and a density-dependent growth of the population. On the other hand, 31] and 32 used time-discrete models classical in populations genetics to show the essential characteristics of the selection dynamics for resistance by one or two insecticides on two autosomal locus. More recently, this line of research was explored again [26, 33. Also, 25] developed genetic models to predict changes in the fitness and frequency of resistance alleles in synergistic scenarios. All these models consider Mendelian inheritance, but do not take into account the existence of several stages of life, and different selective pressure for each phase.

Furthermore, models have been proposed to develop new approaches, such as the idea of evolutionproof insecticides [12, 34, 35]. In particular, these models were developed to explore the control of malaria vector mosquito. Since the malaria parasite requires a mosquito with a long life expectancy, these authors explore strategies to slow down the evolution of resistance using combinations of larvicides and late-life-acting insecticides. In this regard a classical population genetics model is used in 34 to quantify the change in relative frequency of a resistant allele, assuming a constant population of adults. A population genetics model that considers the change in relative frequency of a resistance allele in 
different age classes is considered in [12. The two works cited above differ from [35, in which an agedstructured population of variable size is modeled by delay differential equations. However, this model does not possess inheritance mechanism for autosomal genes.

A non-conventional approach to resistance models was given in [36. Inspired by the study of herbicide resistant weed, this paper proposed a continuous-time deterministic model that combines the logistic growth in a population with the rearrangement of alleles in the genotypes given by inheritance. In addition, the model applies to cases of multiple loci using tensor product, and extends to polyploid and other numbers of alleles. However, it does not allow to model the several life phases, which present different selective pressures in their respective ecological niches.

In 37 a compartmental model with age-structure was proposed to quantify the time required to reverse resistance in a dengue vector mosquito. A model of dimension 15, with five life stages for three genotypes, was introduced, and numerical simulations were conducted, in order to evaluate the loss of resistance, assuming that in absence of insecticide the resistant genotype has lower fitness.

Finally, some recent studies have emphasized the need for models to have an integrated analysis of various control strategies simultaneously. For example, 38 proposed a model for control mosquitoborne diseases including insecticides and Wolbachia, a maternally inherited bacterium with the potential to reduce vector competence. However, the model proposed is limited to an insecticide susceptible mosquito population. Based on empirical evidence, models have been proposed that analyze the synergy between insecticide resistance in chemical control strategies for mosquito epidemics with the use of Wolbachia [14.

\subsection{The proposed modeling approach}

Our goal in the present paper is to provide a class of simple models able to account for the evolution of resistance to larvicides and/or adulticides on an autosomal gene for an insect population, taking into account the life history complexity, and to show that the latter possess key properties that we are entitled to expect from such models. This work is viewed as paving the way towards the construction of more complex ad hoc models, describing more involved situations and useful for various situations involving biological control of vectors. A typical example is the release of Wolbachia infected mosquitoes in presence of, possibly undesired, insecticide. Elaborating and analyzing release strategies for such situations requires a mix of population dynamics, population genetics and control theory. Work built up upon the models and analysis provided here, will be presented in a forthcoming paper.

Insects are diploid organisms with sexual reproduction and usually with several phases of life. In many cases the genes involved in insecticides resistance are autosomal and follow principles of Mendelian inheritance. Furthermore, they have a pre-reproductive and a reproductive phases and quite frequently, like for holometabolism, the immature stages are well differentiated from the mature stages. In such cases the larvae do not compete with adults, since they are found in different ecological niches, and are consequently subjected to different selective pressures - e.g. larvicides and adulticides factors. In response to the aforementioned, we propose:

- a continuous-time compartmental model based on a life history with two leading phases, a prereproductive and reproductive;

- a reproductive phase that includes a heredity function for Mendelian inheritance given by an autosomal gene;

- and selective pressures of larvicides and/or adulticides for each genotype that may affect fertility and density-dependent viability in each life phase.

The life cycle of an insect may be seen analogously to a sequence of chemical modifications that a sustenance goes through. Like in a chemical reaction network 39, some stages of life are slower than others. This last feature opens up the possibility of using slow manifold theory to deduce simpler inheritance models. The modelling framework presented below is based on this principle. More precisely, according to whether the reproductive phase is fast or slow with respect to the pre-reproductive one, we obtain two distinct class of models. This simplification, achieved via slow manifold theory, yields two different classes of Mendelian inheritance models, which present density-dependent recruitment and mortality rates in a non-conventional way. 
We show that these models fulfil several fundamental properties. Most importantly, in the absence of selection they evolve in compliance with Hardy-Weinberg law; while in presence of selection and in the dominant or codominant cases, they globally converge towards the disappearance of all genotypes except the fittest homozygous one. Mendelian inheritance is easily incorporated into population dynamics in the modeling framework proposed here. This is achieved through inheritance matrices deduced from probability principles, allowing for modeling and analysis of the evolution in more complicated situations.

The paper is organized as follows. The modeling framework is presented in Section 2, departing from a general two life phase model. A single locus trait heredity function that formalizes Mendel's first law is described in Section 2.1. In Sections 2.2.1 and 2.2.2 slow manifold theory is used to deduce two classes of models describing the evolution of three genotypes of a population having inheritable attributes and density-dependent recruitment and mortality rates. These two classes correspond respectively to the limit of fast and slow reproductive phase. We extend in Section 3 the latter to two general classes of models, namely $(\mathbf{F})$ (Fast) and $(\mathbf{S})$ (Slow), which are studied afterwards. The assumptions necessary to this study are given in Section 3.1. and useful technical results are put in Section 3.2. Their proofs are in Appendix A Well-posedness of these models and other qualitative results are considered in Section 4.

The asymptotic behavior is then studied. The case where no fitness difference exists between the different genotypes is considered in Section 5. In this case, Hardy-Weinberg law is shown to hold for the considered classes of models (Theorem 13). In Section 6 is studied the case of dominant and codominant selection regimes. Asymptotic convergence to the homozygote with highest fitness is demonstrated in such conditions for models $(\underline{\mathbf{F}})$ and $(\underline{\mathbf{S}})$ (Theorem [14). Last, concluding remarks are made in Section 7

\section{Modelling}

In this section is introduced the approach leading to the models. The latter are introduced in Section 3 and studied afterwards. We begin by listing some of the main constitutive hypotheses made to obtain these models of life history for a diploid population obeying the Mendel's laws of inheritance and submitted to selection.

- No distinction is made between male and female individuals. In particular the fertility and mortality are considered identical for males and females. It is possible to consider this abstraction when the sex ratio is constant and absolute fitness is independent of sex.

- The mortality rates in each life phase are increasing functions of the population density.

- The fertility rates are constant, i.e. they are independent of the population density.

- The differences in fertility or mortality between different genotypes result from an artificial, stationary, modification of the environment e.g. by a constant lethal dose application of an insecticide.

- The differences in inheritable attributes that modify the fitness with respect to natality and mortality are determined by a unique autosomal locus.

- There exist two different types of alleles in this locus, namely $s$ and $r$.

In chemical control, the applied lethal dose of insecticide can vary over time, affecting fertility and mortality in different ways. In the models introduced in the sequel, we do not treat explicitly this time-dependence, and assume that to every given insecticide lethal dose density correspond fixed characteristic fertility and mortality. Once this dependence is modelled, describing intermittent application of insecticide is done by introducing time-dependence of the insecticide density. This aspect is not explored further in this paper, which instead focuses on asymptotic properties in stationary environment.

As a consequence of the setting of two alleles in a single locus of a diploid population, we have three different genotypes, namely:

$$
\{r, s\} \times\{r, s\}=\{(r, r),(s, r),(s, s)\}
$$

as no difference exists between the genotypes $(s, r)$ and $(r, s)$. In the sequel, the index $i=1,2,3$ will be used to identify the genotypes, while generally speaking the index $j=r, s$ will refer to the alleles. The latter are designated $r, s$ as resistant and susceptible. 
As mentioned before, we are interested in the representation of a population with two life phases. This distinction is quite simplistic for insects with complex life cycles, but covers broad distinctions like pre-reproductive phase, subject to food and space limitation, and reproductive phase with different niches and potential selective pressures. As a starting point to represent such life history, we begin with the following compartmental models:

$$
\begin{gathered}
\dot{L}_{i}=\alpha_{i}(F \hat{A}(t))-\mu_{i}\left(v^{\top} L(t)\right) L_{i}(t)-\nu_{i} L_{i}(t) \\
\dot{\hat{A}}_{i}=\nu_{i} L_{i}(t)-\hat{\mu}_{i}\left(\hat{w}^{\top} \hat{A}(t)\right) \hat{A}_{i}(t)
\end{gathered}
$$

for $i=1,2,3$. The quantities $L_{i}$ (Larvae) represent the density of genotypes $(i=1,2,3)$, in prereproductive phase, and $\hat{A}_{i}$ (Adults) the corresponding density of reproductive individuals. The parameters involved in (1) have the following meaning.

- The mortality functions $\mu_{i}$ and $\hat{\mu}_{i}$ depend upon the density, through the use by a population of certain recourse, typically food or space. We assume that these mortality rates are all increasing functions of the density. On the other hand, we wish to allow each genotype to have its proper consumption needs. This is done by introducing weighted sums $v^{\top} L$ and $\hat{w}^{\top} \hat{A}$ as argument of the functions $\mu_{i}$ and $\hat{\mu}_{i}$, for given positive vectors $v, \hat{w}$.

- The functions $\alpha_{i}$ account for the mechanism of Mendelian inheritance, presented in detail in Section 2.1. while, for positive $f_{i}, i=1,2,3$, the diagonal matrix $F:=\operatorname{diag}\left\{f_{i}\right\}$ represents multiplicative symmetry fertility rates of genotypes [40. The "symmetry" here refers to the fact that the numbers of births resulting from a given genotypic crossing $i \times i^{\prime}$ are identical whether females carry the genotype $i$ and males the genotype $i^{\prime}$ or the opposite. Modeling of non-symmetric fertilities of mating pairs in one-locus genetics yields quite complicated models [17].

- Last, the $\nu_{i}$ describe constant maturation rates from pre-reproductive to reproductive phase.

Defining $A:=F \hat{A}$, that is $A_{i}:=f_{i} \hat{A}_{i}$, system (1) rewrites as

$$
\begin{gathered}
\dot{L}_{i}=\alpha_{i}(A(t))-\mu_{i}\left(v^{\top} L(t)\right) L_{i}(t)-\nu_{i} L_{i}(t) \\
\dot{A}_{i}=\hat{\nu}_{i} L_{i}(t)-\hat{\mu}_{i}\left(w^{\top} A(t)\right) A_{i}(t),
\end{gathered}
$$

for $i=1,2,3$, where by definition

$$
\hat{\nu}_{i}:=f_{i} \nu_{i}, \quad w_{i}=f_{i}^{-1} \hat{w}_{i}
$$

\subsection{Single locus trait inheritance}

We present in this section the heredity functions $\alpha_{i}$ used to model Mendelian inheritance. As already said, in this paper we consider a general diploid population composed of three genotypes and assume no distinction between male and female individuals.

We associate in the sequel to $A_{1}(t)$ (resp. $A_{2}(t)$, resp. $\left.A_{3}(t)\right)$ the density of individuals with genotype $(r, r)$ (resp. $(r, s)$, resp. $(s, s))$ at time $t$ in the population: the two homozygous genotypes are represented by $A_{1}$ and $A_{3}$, while $A_{2}$ represents the heterozygous genotype.

Considering all possible crosses given random mating, the expected frequency of two allele combinations in a diploid population is obtained from a Punnett Square [4] and is represented in the following table:

\begin{tabular}{c|c|ccc} 
Offspring & $\sigma^{r} \backslash q$ & $A_{1}(r, r)$ & $A_{2}(r, s)$ & $A_{3}(s, s)$ \\
\hline \multirow{4}{*}{$(r, r)$} & $A_{1}$ & 1 & $1 / 2$ & 0 \\
& $A_{2}$ & $1 / 2$ & $1 / 4$ & 0 \\
& $A_{3}$ & 0 & 0 & 0 \\
\hline \multirow{4}{*}{$(r, s)$} & $A_{1}$ & 0 & $1 / 2$ & 1 \\
& $A_{2}$ & $1 / 2$ & $1 / 2$ & $1 / 2$ \\
& $A_{3}$ & 1 & $1 / 2$ & 0 \\
\hline \multirow{3}{*}{$(s, s)$} & $A_{1}$ & 0 & 0 & 0 \\
& $A_{2}$ & 0 & $1 / 4$ & $1 / 2$ \\
& $A_{3}$ & 0 & $1 / 2$ & 1 \\
\hline
\end{tabular}


Defining the vectors

$$
u_{r}:=\left(\begin{array}{c}
1 \\
\frac{1}{2} \\
0
\end{array}\right), \quad u_{s}:=\left(\begin{array}{c}
0 \\
\frac{1}{2} \\
1
\end{array}\right), \quad \mathbf{1}:=\left(\begin{array}{l}
1 \\
1 \\
1
\end{array}\right)=u_{r}+u_{s}
$$

the relative frequency of the genotype $i, i=1,2,3$, in the offspring produced by the population density $A$ is given by

$$
\frac{\left(u_{r}^{\top} A\right)^{2}}{\left(\mathbf{1}^{\top} A\right)^{2}}, \quad 2 \frac{\left(u_{r}^{\top} A\right)\left(u_{s}^{\top} A\right)}{\left(\mathbf{1}^{\top} A\right)^{2}}, \quad \frac{\left(u_{s}^{\top} A\right)^{2}}{\left(\mathbf{1}^{\top} A\right)^{2}} .
$$

Making the normalization hypothesis that the total density of offsprings (of all genotypes) hatched by time unit equals the total density of adults of all genotypes, that is $\alpha_{1}(A)+\alpha_{2}(A)+\alpha_{3}(A)=A_{1}+A_{2}+A_{3}$ for any $A \in \mathbb{R}_{+}^{3}$, their genotypic density repartition is therefore given by:

$$
\alpha_{1}(A):=\frac{\left(u_{r}^{\top} A\right)^{2}}{\mathbf{1}^{\top} A}, \quad \alpha_{2}(A):=2 \frac{\left(u_{r}^{\top} A\right)\left(u_{s}^{\top} A\right)}{\mathbf{1}^{\top} A}, \quad \alpha_{3}(A):=\frac{\left(u_{s}^{\top} A\right)^{2}}{\mathbf{1}^{\top} A},
$$

or equivalently

$$
\alpha_{i}(A):=\frac{1}{\mathbf{1}^{\top} A} A^{\top} G_{i} A, \quad i=1,2,3,
$$

with inheritance matrices $G_{i}, i=1,2,3$, defined by

$G_{1}=u_{r} u_{r}^{\top}=\left(\begin{array}{ccc}1 & 1 / 2 & 0 \\ 1 / 2 & 1 / 4 & 0 \\ 0 & 0 & 0\end{array}\right), G_{2}=u_{r} u_{s}^{\top}+u_{s} u_{r}^{\top}=\left(\begin{array}{ccc}0 & 1 / 2 & 1 \\ 1 / 2 & 1 / 2 & 1 / 2 \\ 1 & 1 / 2 & 0\end{array}\right), G_{3}=u_{s} u_{s}^{\top}=\left(\begin{array}{ccc}0 & 0 & 0 \\ 0 & 1 / 4 & 1 / 2 \\ 0 & 1 / 2 & 1\end{array}\right)$.

We also define the operator $\alpha: \mathbb{R}_{+}^{3} \backslash\left\{0_{3}\right\} \rightarrow \mathbb{R}_{+}^{3} \backslash\left\{0_{3}\right\}$ as

$$
\alpha(A):=\left(\begin{array}{c}
\alpha_{1}(A) \\
\alpha_{2}(A) \\
\alpha_{3}(A)
\end{array}\right)
$$

Coming back to the initial setting of (2a), one sees that the expected frequency of the zygotes depend upon the crossings, through the diagonal matrix $F$ multiplying the unnormalized vector of adult densities $\hat{A}$. This setting represents indeed symmetric multiplicative fertility [40].

A quite similar matrix setting was introduced by Langemann et al. [36. to model the evolution of herbicide resistance, in the case of single and multiple loci. It is well fitted to this purpose, with the $G_{i}$ matrix materializing the inheritance mechanisms expressed by the first Mendel's Law (Law of Segregation of genes). Notice also that the outer product between the probability vectors $u_{j} u_{j^{\prime}}^{\top}$ corresponds to the encounters of a sex with the opposite. Other inheritance mechanisms may be modelled in this framework, including mechanisms that involve multiple loci [36].

\subsection{Fast-slow models of population dynamics}

The life history of organisms in general and insect in particular is quite different from one to the other. Some mature early and reproduce quickly, while others mature late and reproduce slowly. An extreme example is the arachnids Adactylidium sp., which are born mature and, having hatched inside their mother, mate with their brothers 42, 43. The insects Ephemeroptera constitute another extreme case: the life of an adult Mayfly is very short and has essentially the primary function of reproduction 44.

We consider in the sequel the cases where one of the life phases is sensibly faster than the other one, in other words that a fast dynamics and a slow dynamics are present in (2). In such conditions, it is reasonable to expect that the fast variable in (2) should evolve rapidly and reach a unique equilibrium value, depending upon the value of the slow variable, as if the latter was frozen; and that the global evolution could be described by the evolution of the slow variable alone, taking for the fast one the previous equilibrium value. This heuristic, consisting in approximating the initial fast-slow system by a smaller slow system, may indeed be firmly justified, by applying singular perturbation methods [45. The latter simplifies the dimension of the system analyzed and preserves the parameters and properties of 
the original system. Under adequate assumptions, when the equilibrium value is asymptotically stable, then the approximation holds on any finite time interval, and even on infinite time intervals when the slow system has an asymptotically stable equilibrium, see e.g. [46, 47] for details.

Depending on which of the phases is faster, applying singular perturbation yields two different classes of models. We show in Sections 2.2.1 (fast reproductive phase) and 2.2.2 (slow reproductive phase) how these two classes are obtained.

\subsubsection{Fast reproductive phase population dynamics}

We consider here the case of an organism with a reproductive phase faster than the pre-reproductive phase. As said before, the mortality rates are increasing, and this is specially true for $\hat{\mu}_{i}$ in (2b). Therefore, for any fixed $L$, equation (2b) possesses a unique, globally asymptotically stable, equilibrium $A(L)$, given implicitly by

$$
0=\hat{\nu}_{i} L_{i}-\hat{\mu}_{i}\left(w^{\top} A\right) A_{i}, \quad i=1,2,3 .
$$

Equation (86) yields the following algebraic relationship between $L_{i}$ and $A_{i}$ :

$$
A_{i}=\frac{\hat{\nu}_{i}}{\hat{\mu}_{i}\left(w^{\top} A\right)} L_{i}, \quad i=1,2,3
$$

which necessarily implies that $b:=w^{\top} A$ fulfills the identity

$$
b=\sum_{i=1}^{3} \frac{w_{i} \hat{\nu}_{i}}{\hat{\mu}_{i}(b)} L_{i}
$$

Now, the mortality $\hat{\mu}_{i}$ is increasing with the total population, so for all $L \in \mathbb{R}_{+}^{3} \backslash\{0\}$, the map

$$
b \mapsto \sum_{i=1}^{3} \frac{w_{i} \hat{\nu}_{i}}{\hat{\mu}_{i}(b)} L_{i}
$$

is decreasing and may be inverted, providing a unique solution, denoted $b^{*}(L)$, to equation (9). For any given nonnegative vector $L$, the unique solution of (8) is then given as

$$
A_{i}=\frac{\hat{\nu}_{i}}{\hat{\mu}_{i}\left(b^{*}(L)\right)} L_{i}, \quad i=1,2,3
$$

In the limiting case where the duration of the mature phase is sensibly faster than the immature one, one may approximate equation (2) through slow manifold theory [45, and the asymptotic evolution is then expressed by the algebro-differential system

$$
\dot{L}_{i}=\alpha_{i}(A)-\mu_{i}\left(v^{\top} L\right) L_{i}-\nu_{i} L_{i}, \quad A_{i}=\frac{\hat{\nu}_{i}}{\hat{\mu}_{i}\left(b^{*}(L)\right)} L_{i}, \quad i=1,2,3 .
$$

Defining for any nonnegative scalar $b, m_{i}(b):=\frac{\hat{\nu}_{i}}{\hat{\mu}_{i}(b)}$, one then ends up with the system

$$
\dot{L}_{i}=\alpha_{i}\left(\operatorname{diag}\left\{m_{i}\left(b^{*}(L)\right)\right\} L\right)-\left(\nu_{i}+\mu_{i}\left(v^{\top} L\right)\right) L_{i}, \quad i=1,2,3
$$

where, for any $L \in \mathbb{R}_{+}^{3}, b^{*}(L)$ is defined implicitly by the identity:

$$
b^{*}(L)=\sum_{i=1}^{3} w_{i} m_{i}\left(b^{*}(L)\right) L_{i} .
$$

In (13a), $\operatorname{diag}\left\{m_{i}\left(b^{*}(L)\right)\right\}$ denotes the diagonal matrix formed with the scalar coefficients $m_{i}\left(b^{*}(L)\right)$, $i=1,2,3$. Notice that by construction the functions $m_{i}$ present in (13) are decreasing. The scalar $b^{*}(L)$ represents the weighted density of adults $w^{\top} A$ corresponding to steady-state population $L$ in prereproductive stage. There is no difficulty in expressing the functions and coefficients of system (13) in terms of the initial problem (1), through the above formulas and the identities in (3). 


\subsubsection{Slow reproductive phase population dynamics}

Consider now the opposite situation, of an reproductive phase comparatively much slow than the prereproductive one. In this case, the same argument than before leads similarly to consider instead of system (2) the algebro-differential model:

$$
0=\alpha_{i}(A)-\mu_{i}\left(v^{\top} L\right) L_{i}-\nu_{i} L_{i}, \quad \dot{A}_{i}=\hat{\nu}_{i} L_{i}-\hat{\mu}_{i}\left(w^{\top} A\right) A_{i}, \quad i=1,2,3
$$

The algebraic relationship in equation (14) provides the identities

$$
L_{i}=\frac{1}{\nu_{i}+\mu_{i}\left(v^{\top} L\right)} \alpha_{i}(A), \quad i=1,2,3
$$

and thus $b:=v^{\top} L$ necessarily fulfils the condition:

$$
b=\sum_{i=1}^{3} \frac{v_{i}}{\nu_{i}+\mu_{i}(b)} \alpha_{i}(A)
$$

From the increasingness of the mortality functions, one deduces as before that, for all $A \in \mathbb{R}_{+}^{3} \backslash\{0\}$, the right-hand side of (16) is decreasing, and this relation may thus be inverted. This operation yields a unique solution to equation (16), which is denoted $b^{*}(\alpha(A))$, using $\alpha$ defined in (7). With this, for any nonnegative $A$, (15) has a unique solution $L(A)$, which writes

$$
L_{i}=\frac{1}{\nu_{i}+\mu_{i}\left(b^{*}(\alpha(A))\right)} \alpha_{i}(A), i=1,2,3 .
$$

Defining here for any nonnegative scalar $b,\left(\right.$ decreasing) functions $m_{i}(b):=\frac{\hat{\nu}_{i}}{\nu_{i}+\mu_{i}(b)}$, one obtains finally the model:

$$
\dot{A}_{i}=\alpha_{i}(A) m_{i}\left(b^{*}(\alpha(A))\right)-\hat{\mu}_{i}\left(w^{\top} A\right) A_{i}, \quad i=1,2,3
$$

where, for any $A \in \mathbb{R}_{+}^{3}, b^{*}(A)$ is defined implicitly by

$$
b^{*}(A)=\sum_{i=1}^{3} \frac{v_{i}}{\hat{\nu}_{i}} m_{i}\left(b^{*}(A)\right) A_{i}
$$

Equation (17) is quite similar to, but different from, equation (13) obtained in the case of a fast reproductive phase. Here, the scalar $b^{*}(A)$ is the weighted density $w^{\top} L$ of individuals in the pre-reproductive phase that corresponds to steady-state population $A$ in reproductive stage. As in Section 2.2.1, there is no specific difficulty here in expressing the functions and coefficients of system (17) in terms of the initial problem (1), using the above transformations and the identities in (3).

As can be seen from the derivations of (13) and (17), monotonicity assumptions on the mortality rates are necessary, in order to obtain the perturbed systems. For this reason, we postpone the formal statement of the models studied in the sequel to Section 3, where all the assumptions are introduced.

\section{Preliminaries}

\subsection{Assumptions}

We now introduce the two general classes of equations studied in this paper. The latter extend in particular the systems (13) and (17) obtained previously as models for fast and slow reproductive phase populations. The notations are inspired from these preliminary examples and generalised.

We introduce first functions $m_{i}$ and $\mu_{i}$ to model recruitment and mortality rates, and $v, w$ two vectors permitting to define their arguments. The following series of assumptions will be made on these objects.

Assumption 1. For any $i=1,2,3$, the functions $m_{i}: \mathbb{R}_{+} \rightarrow \mathbb{R}_{+}$are locally Lipschitz decreasing functions; the functions $\mu_{i}: \mathbb{R}_{+} \rightarrow \mathbb{R}_{+}$are locally Lipschitz increasing functions. The vectors $v, w \in \mathbb{R}^{3}$ have positive components. 
Assumption [1 assumes that the recruitment rates are decreasing functions, while the mortality rates are increasing functions, as is the case for the Verhulst logistic equation and other conventional population dynamics models. As a central consequence, this allows to give sense to the function $b^{*}$ that naturally appeared in Sections 2.2.1 and 2.2.2, as shown by the following result, whose proof is in Appendix A.1 The values $v_{i}$ that appear in the statement are the components of the vector $v$ introduced in Assumption 1.

Lemma 1. Let Assumption 1 holds. Then, for any $x \in \mathbb{R}_{+}^{3}$, there exists a unique solution $b^{*}(x) \in \mathbb{R}_{+}$, to the scalar equation

$$
b=\sum_{i=1}^{3} v_{i} m_{i}(b) x_{i} .
$$

Moreover, $b^{*}(x)>0$ if $x \in \mathbb{R}_{+}^{3} \backslash\left\{0_{3}\right\}, b^{*}\left(0_{3}\right)=0$ and $b^{*}: \mathbb{R}_{+}^{3} \rightarrow \mathbb{R}_{+}$has the same regularity than the functions $m_{i}$.

As explained in Sections 2.2.1 and 2.2.2 for any density $x \in \mathbb{R}_{+}^{3} \backslash\left\{0_{3}\right\}$, the scalar $b^{*}(x)$ in Lemma 1 represents the total effective density of individuals present in the other life stage, reached here infinitely fast. Notice that the map $b^{*}$ depends upon the vector $v$.

With this done, we are finally in position to introduce the two classes of models studied in this paper. For any $x \in \mathbb{R}_{+}^{3}$ and $z \in \mathbb{R}_{+}$, define the positive diagonal matrices $M(x)$ and $\mu(z)$ by:

$$
M(x)=\operatorname{diag}\left\{m_{i}\left(b^{*}(x)\right)\right\}, \quad \mu(z)=\operatorname{diag}\left\{\mu_{i}(z)\right\} .
$$

The two classes of models we will be interested in are expressed as follows:

$$
\begin{gathered}
\dot{x}=\alpha(M(x) x)-\mu\left(w^{\top} x\right) x, \\
\dot{x}=M(\alpha(x)) \alpha(x)-\mu\left(w^{\top} x\right) x .
\end{gathered}
$$

Equations $(\mathbf{F}$ ) and $(\mathbf{S})$ can be described as a class of inheritance models where population dynamics is governed by density-dependent recruitment and mortality. The first terms in the right hand side of these equations models recruitment, while the second term models mortality. Conventional population dynamics models implicitly assume that all individuals have on average the same fertility and viability. However, it is clear that this condition may vary according to heritable traits, e.g. resistance to an insecticide. The class of inheritance models proposed generalizes a conventional population dynamics system for the case of three genotypes governed by a locus, such that selective pressure can affect recruitment or mortality.

Recall that the map $\alpha$ is defined in (7). In developed form, these equations write respectively

$$
\begin{aligned}
& \dot{x}_{1}=\frac{\left(u_{r}^{\top} M(x) x\right)^{2}}{\mathbf{1}^{\top} M(x) x}-\mu_{1}\left(w^{\top} x\right) x_{1} \\
& \dot{x}_{2}=2 \frac{\left(u_{r}^{\top} M(x) x\right)\left(u_{s}^{\top} M(x) x\right)}{\mathbf{1}^{\top} M(x) x}-\mu_{2}\left(w^{\top} x\right) x_{2} \\
& \dot{x}_{3}=\frac{\left(u_{s}^{\top} M(x) x\right)^{2}}{\mathbf{1}^{\top} M(x) x}-\mu_{3}\left(w^{\top} x\right) x_{3}
\end{aligned}
$$

and

$$
\begin{aligned}
& \dot{x}_{1}=\frac{\left(u_{r}^{\top} x\right)^{2}}{\mathbf{1}^{\top} x} m_{1}\left(b^{*}(\alpha(x))\right)-\mu_{1}\left(w^{\top} x\right) x_{1} \\
& \dot{x}_{2}=2 \frac{\left(u_{r}^{\top} x\right)\left(u_{s}^{\top} x\right)}{\mathbf{1}^{\top} x} m_{2}\left(b^{*}(\alpha(x))\right)-\mu_{2}\left(w^{\top} x\right) x_{2} \\
& \dot{x}_{3}=\frac{\left(u_{s}^{\top} x\right)^{2}}{\mathbf{1}^{\top} x} m_{3}\left(b^{*}(\alpha(x))\right)-\mu_{3}\left(w^{\top} x\right) x_{3} .
\end{aligned}
$$

Manifestly, equations $(\underline{\mathbf{F}})$ and $(\underline{\mathbf{S}})$ contain as particular cases the equations (13) and (17), obtained by applying singular perturbation to the normalized system (11). One shows easily that the system (13) that 
emerged in the case of fast reproductive phase (Section 2.2.1) is system (F) with the recruitment rates $m_{i}$ and the mortality rates $\mu_{i}$

$$
\frac{f_{i} \nu_{i}}{\hat{\mu}_{i}(\cdot)} \quad \text { and } \quad \nu_{i}+\mu_{i}(\cdot) \text {. }
$$

The variable $x$ represents the pre-adult populations in the original model. On the other hand, system (17) appeared in the slow reproductive phase (Section 2.2.2) corresponds to (S) with recruitment and mortality rates

$$
\frac{f_{i} \nu_{i}}{\nu_{i}+\mu_{i}(\cdot)} \quad \text { and } \quad \hat{\mu}_{i}(\cdot)
$$

and $x$ now represents the adult populations. Expressed in terms of the coefficients of the starting model (11), the recruitment rate in systems (13) and (17) depends jointly upon the genotype fertility $f_{i}$, the maturation rate $\nu_{i}$ and the density-dependent mortality in the corresponding life phase.

The population dynamics under selective pressure (in adaptation) implies a difference in fitness between genetically distinct individuals, either in the recruitment or mortality rate. The following supplementary assumptions will be used to study adaptation.

Assumption 2. The functions $m_{i}, \mu_{i}$ satisfy

$$
\forall x \in \mathbb{R}_{+}^{3}, \quad m_{1}\left(b^{*}(x)\right) \geq m_{2}\left(b^{*}(x)\right) \geq m_{3}\left(b^{*}(x)\right) \quad \text { and } \quad \forall z \geq 0, \quad \mu_{1}(z) \leq \mu_{2}(z) \leq \mu_{3}(z)
$$

Moreover, for any $x \in \mathbb{R}_{+}^{3}$,

- $m_{1}\left(b^{*}(x)\right)-m_{3}\left(b^{*}(x)\right)+\mu_{3}\left(w^{\top} x\right)-\mu_{1}\left(w^{\top} x\right)>0$ for system $(\mathbf{F}) ;$

- $m_{1}\left(b^{*}(\alpha(x))\right)-m_{3}\left(b^{*}(\alpha(x))\right)+\mu_{3}\left(w^{\top} x\right)-\mu_{1}\left(w^{\top} x\right)>0$ for system (S).

Assumption 3. The following inequality holds

$$
m_{1}(0)>\mu_{1}(0)
$$

Assumption 4. For any $x \in \mathbb{R}_{+}^{3} \backslash\left\{0_{3}\right\}$, the following limits exist and satisfy

$$
0 \leq \lim _{\lambda \rightarrow+\infty} m_{i}(\lambda x)<\lim _{\lambda \rightarrow+\infty} \mu_{i}(\lambda) \leq+\infty, \quad i=1,2,3 .
$$

Assumption 2 imposes a relative ordering of the fitnesses of the three genotypes, associated with corresponding increasing mortality rates or decreasing recruitment rates. Two distinct important situations are covered: the cases of codominance or incomplete dominance, when the fitness of the two homozygotes and of the heterozygote are strictly ordered according to (19) - e.g. $m_{1}>m_{2}>m_{3}$ and $\mu_{1}<\mu_{2}<\mu_{3}$ ); and the case of dominance, where the heterozygote has the same fitness than one of the two homozygotes - e.g. $m_{1}=m_{2}=m_{3}$, and $\mu_{1}<\mu_{2}=\mu_{3}$ or $\mu_{1}=\mu_{2}<\mu_{3}$; or $\mu_{1}=\mu_{2}=\mu_{3}$, and $m_{1}>m_{2}=m_{3}$ or $m_{1}=m_{2}>m_{3}$. Notice that the selectively neutral case, where the fitness of all genotypes is equal, is excluded by the positivity requirement contained in Assumption 2 .

Assumption 3 ensures that at least genotype 1, with highest fitness, is viable. Last, Assumption 4 indicates that in any sufficiently large population, the mortality rates are greater than the recruitment rates for each genotype, and this overpopulation effect will limit the population growth.

\subsection{Technical lemmas}

Before proceeding to the analysis results in Section 4 we state now two technical lemmas useful in the sequel. By definition, $e_{i}, i=1,2,3$, represents the $i$ th unit vector of $\mathbb{R}^{3}$.

Lemma 2. For any $x \in \mathbb{R}_{+}^{3} \backslash\left\{0_{3}\right\}$ and any $\lambda>0$, the following properties are fulfilled
1. $u_{j}^{\top} \alpha(x)=u_{j}^{\top} x, j=r, s$;
2. $\mathbf{1}^{\top} \alpha(x)=\mathbf{1}^{\top} x$;
3. $\alpha(\lambda x)=\lambda \alpha(x)$;
4. $\alpha\left(e_{i}\right)=e_{i}, i=1,3$. 
Recall that the map $\alpha$ is defined in (7), and that the vectors $u_{r}, u_{s}$ and $\mathbf{1}$ come from (4). We explain now the meaning of these properties. Point 1 manifests that the density of each allele hatched per time unit is equal to its density, as a consequence of the mechanisms of Mendelian inheritance, and therefore the same property holds for the global population (Point 2). This is a consequence of the normalization exposed in Section 2.1. Point 3 indicates that the operator $\alpha$ is homogeneous of degree 1: the zygote repartition in the offspring only depends upon the zygotic proportion of adults, while the offspring birth density is proportional to the adult density. Last, Point 4 states that a homogeneous population of one of the homozygotes only gives birth to homozygotes of the same genotype.

Lemma 3. Let $x \in \mathbb{R}_{+}^{3} \backslash\{0\}$.

1. The following properties are satisfied

$$
\begin{gathered}
\lim _{\lambda \rightarrow+\infty} b^{*}(\lambda x)=+\infty, \\
\lim _{\lambda \rightarrow+\infty} m_{i}\left(b^{*}(\lambda x)\right)<\lim _{\lambda \rightarrow+\infty} \mu_{i}(\lambda), \quad i=1,2,3 .
\end{gathered}
$$

Moreover, for both limits the convergence is uniform in the set $\left\{x \in \mathbb{R}_{+}^{3}: w^{\top} x=1\right\}$.

2. The map $\lambda \mapsto b^{*}(\lambda x)$ is increasing on $[0,+\infty)$.

Proofs of Lemmas 2 and 3 are given in Appendices A.2 and A.3. Point 1 indicates that, for any population $x$ of the slow phase, the population $b^{*}(\lambda x)$ of the fast phase grows unbounded when $\lambda$ goes to infinity; and that, the birth rate $m_{i}\left(b^{*}(\lambda x)\right)$ in the slow life stage $i$ is smaller than the mortality rate $\mu_{i}(\lambda)$ when $\lambda$ goes to infinity. Both these properties are uniform with respect to the genotypic repartition in the population $x$. Point 1 states that the map, for given slow phase population $x$, the fast phase population $b^{*}(\lambda x)$ increases with $\lambda$. Notice however that the map $x \mapsto b^{*}(x)$ itself is not increasing.

\section{Well-posedness and qualitative results}

We provide here the first results concerning the solutions of systems $(\underline{\mathbf{F}})$ and $(\underline{\mathbf{S}})$. At first, well-posedness of these systems of nonlinear ordinary differential equations is proved in Section 4.1. The qualitative properties that relate to the existence of monomorphic and polymorphic states, and trajectories are studied in section 4.2. Last, we show in Section 4.3 that quite natural notions of mean allelic recruitment rate and mean allelic mortality rate may be introduced for each system, which will prove quite useful in establishing the forthcoming asymptotic results.

\subsection{Well-posedness of the models}

Well-posedness for the models $(\underline{\mathbf{F}})$ and $(\underline{\mathbf{S}})$ is supplied by the following statement.

Theorem 4 (Well-posedness and boundedness of the solutions). Assume Assumption 1 is fulfilled. Then, for any nonnegative initial condition, there exists a unique solution $x$ of $(\mathbf{F})$, resp. $(\mathbf{S})$, on $[0,+\infty)$, continuous with respect to the initial condition. Its coordinates are nonnegative for any $t \geq 0$.

If moreover Assumption 4 is fulfilled, then

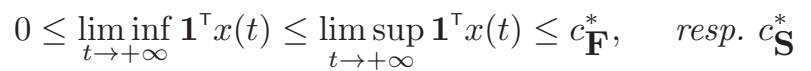

where by definition $q_{\mathbf{F}}^{*}, \operatorname{resp} . q_{\mathbf{S}}^{*}$, is the largest $c \geq 0$ such that

$$
\begin{array}{r}
\max \left\{\max _{i} \frac{m_{i}\left(b^{*}(c x)\right)}{\mu_{i}(c)}: w^{\top} x=1\right\} \leq 1, \\
\text { resp. } \max \left\{\max _{i} \frac{m_{i}\left(b^{*}(c \alpha(x))\right)}{\mu_{i}(c)}: w^{\top} x=1\right\} \leq 1
\end{array}
$$


Formula (24) guarantees that the unique solution corresponding to a given initial condition, takes on nonnegative values and is asymptotically bounded from above by a constant value, independent of the trajectory. Inequality (25) provides an estimate of this bound. This differs from the conventional dynamics for Mendelian populations continuous model — see Chapters 19 and 22 in [17, in which the population is governed by a Malthusian fitness and goes exponentially towards infinity together with all alleles of the gene pool.

Proof.

- The equations $(\mathbf{F})$ and $(\mathbf{S})$ are meaningful as soon as Assumption 1 holds, as the latter permits to define $b^{*}$ (through Lemma 1). The well-posedness of both systems comes from the Lipschitzness of their right-hand side. The fact that the trajectories do not escape the nonnegative quadrant comes from the fact that $\dot{x}_{i}(t) \geq 0$ whenever $x_{i}(t)=0, i=1,2,3$.

- We first demonstrate that the definition of $q_{\mathbf{F}}^{*}$ as stated in the statement is meaningful. Indeed, for any $x \in \mathbb{R}_{+}^{3} \backslash\left\{0_{3}\right\}$, due to Lemma 3 the map

$$
c \mapsto \max _{i=1,2,3} \frac{m_{i}\left(b^{*}(c x)\right)}{\mu_{i}(c)}
$$

is decreasing and, due to Assumption 4, it satisfies:

$$
\lim _{c \rightarrow+\infty} \max _{i=1,2,3} \frac{m_{i}\left(b^{*}(c x)\right)}{\mu_{i}(c)}<1 .
$$

As the convergence of this limit is uniform in the set $\left\{x \in \mathbb{R}_{+}^{3}: w^{\top} x=1\right\}$, the map

$$
c \mapsto \max \left\{\max _{i=1,2,3} \frac{m_{i}\left(b^{*}(c x)\right)}{\mu_{i}(c)}: w^{\top} x=1\right\}
$$

admits values smaller than 1 for sufficiently large $c>0$. Therefore, $q_{\mathbf{F}}^{*}$ as given in the statement is well defined, too.

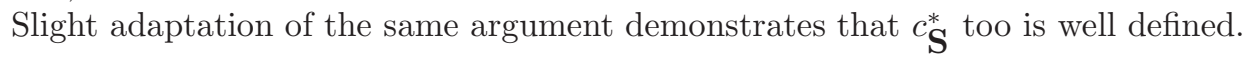

- Summing up the three equations in $(\underline{\mathbf{F}})$, we have

$$
\begin{aligned}
\mathbf{1}^{\top} \dot{x} & =\mathbf{1}^{\top} \alpha(M(x) x)-\mathbf{1}^{\top} \mu\left(w^{\top} x\right) x \\
& =\mathbf{1}^{\top} M(x) x-\mathbf{1}^{\top} \mu\left(w^{\top} x\right) x \quad \text { (by Property 2 in Lemma 2) } \\
& =\sum_{i=1}^{3}\left(m_{i}\left(b^{*}(x)\right)-\mu_{i}\left(w^{\top} x\right)\right) x_{i} \\
& \leq\left(\max _{i}\left\{m_{i}\left(b^{*}(x)\right)-\mu_{i}\left(w^{\top} x\right)\right\}\right) \mathbf{1}^{\top} x, \quad i=1,2,3 .
\end{aligned}
$$

The vector $\frac{x}{w^{\top} x}=1$ is normalised, in the sense that $w^{\top} \frac{x}{w^{\top} x}=1$. As $m_{i}\left(b^{*}(x)\right)=m_{i}\left(b^{*}\left(w^{\top} x \frac{x}{w^{\top} x}\right)\right)$, one gets by definition of $q_{\mathbf{F}}^{*}$ that $\max _{i}\left\{m_{i}\left(b^{*}(x)\right)-\mu_{i}\left(w^{\top} x\right)\right\}<0$ whenever $w^{\top} x>q_{\mathbf{F}}^{*}$.

Essentially the same argument provides the corresponding result for system (S) , and this proves (24) and (25), and achieves the demonstration of Theorem 4

\subsection{Monomorphism and polymorphism}

Variability is essential for the selection to operate in a population. With this in view, we introduce some related notions.

Definition 5 (Monomorphic, polymorphic and "holomorphic" states). A nonzero population state $x \in$ $\mathbb{R}_{+}^{3}$ is called monomorphic if it consists of a single homozygous genotype, that is $x=c e_{i}$ for $i=1$ or 3 and a certain $c>0$. Otherwise it is called polymorphic, and "holomorphic" if all the genotypes are present.

We exhibit now the possible values of a homozygous equilibrium point. 
Lemma 6 (Monomorphic equilibria). Assume Assumptions 1 and 1 are fulfilled. For any $i \in\{1,3\}$, $c_{i}^{*} e_{i}$ is a monomorphic equilibrium, where

- If $m_{i}(0)>\mu_{i}(0), c_{i}^{*} \in \mathbb{R}_{+}$is the unique positive solution to the scalar equation

$$
m_{i}\left(b^{*}\left(c_{i} e_{i}\right)\right)=\mu_{i}\left(c_{i} w_{i}\right) .
$$

- If $m_{i}(0) \leq \mu_{i}(0)$, we let $c_{i}^{*}:=0$.

Recall that $e_{i}$ denotes the $i$ th unit vector.

Proof. Notice that the functions defined on $\mathbb{R}^{+}$by $c \mapsto m_{i}\left(b^{*}\left(c e_{i}\right)\right), i=1,2,3$, are decreasing, due to Assumption 1 and property 1 in Lemma 3 while the functions $c \mapsto \mu_{i}\left(c w_{i}\right), i=1,2,3$, are increasing. Therefore for $m_{i}(0)>\mu_{i}(0)$, (26) admits a unique, positive, solution.

The following lemma shows that the trajectories of $(\mathbf{F})$ and $(\underline{\mathbf{S}})$ initially originated from a monomorphic population converge to the corresponding monomorphic equilibrium.

Lemma 7 (Monomorphic trajectories). Assume Assumptions 1 and 4 are fulfilled. Then any trajectory originating from a monomorphic state, say of homozygote $i \in\{1,3\}$, stays monomorphic and converges towards the corresponding equilibrium point $c_{i}^{*} e_{i}$, with $c_{i}^{*}$ given by Lemma 6 .

The positive numbers $c_{i}^{*}$ represent the carrying capacities corresponding to each monomorphic homozygous population.

Proof. Clearly when only one allele is initially present, i.e. when the heterozygous genotype and one of the two homozygous ones are initially absent, this property remains true throughout time. The dynamics of $(\mathbf{F})$ or $(\underline{\mathbf{S}})$ then occurs in the one-dimensional space that corresponds to this homozygous genotype, and it is easily shown that the evolution obeys the law:

$$
\dot{x}_{i}=\left(m_{i}\left(b^{*}\left(x_{i} e_{i}\right)\right)-\mu_{i}\left(w_{i} x_{i}\right)\right) x_{i} .
$$

In the case where $c_{i}^{*}$ is such that $m_{i}\left(b^{*}\left(c_{i}^{*} e_{i}\right)\right)-\mu_{i}\left(w_{i} c_{i}^{*}\right)=0$, that is when $i=1$, or when $i=3$ and $m_{3}(0)>\mu_{3}(0)$, then $c_{i}^{*}$ is the unique globally asymptotically stable equilibrium of this system.

When $m_{3}(0) \leq \mu_{3}(0)$, all trajectories converge towards the globally asymptotically equilibrium $0=$ $c_{3}^{*} e_{3}$, as in this case $c_{3}^{*}=0$.

We now consider trajectories of $(\mathbf{E})$ and $(\underline{\mathbf{S}})$ such that $u_{r}^{\top} x(0) \neq 0$ and $u_{s}^{\top} x(0) \neq 0$. The following result shows that in such cases all alleles, but also all genotypes, are present for positive times: extinction of a genotype (in the selection case) may only occur asymptotically in time. In other words, polymorphic trajectories are also "holomorphic" trajectories: immediately after the initial time, they contain all genotypes.

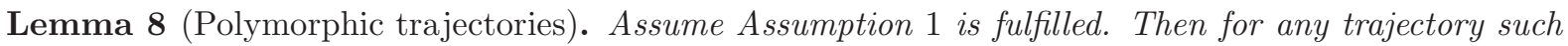
that $u_{r}^{\top} x(0) \neq 0$ and $u_{s}^{\top} x(0) \neq 0$,

$$
\forall t>0, \quad x_{i}(t)>0, \quad i=1,2,3 .
$$

Proof of Lemma 8 If $x_{i}(0)=0$ for $i=1$ or 3 , and $x_{2}(0)>0$, then the corresponding derivative $\dot{x}_{i}$ is positive at time $t=0$, and thus $x_{i}$ takes on positive values at the right of 0 . Similarly, if $x_{i}(0)>0$ for $i=1$ and 3 , and $x_{2}(0)=0$, the same occurs for the derivative $\dot{x}_{2}$, and $x_{2}$ is also positive at the right of 0 .

On the other hand, one sees that, for any $t \geq 0$ and $i=1,2,3$,

$$
\dot{x_{i}} \geq-\mu_{i}\left(w^{\top} x\right) x_{i} .
$$

Therefore

$$
\forall t \geq t^{\prime} \geq 0, x_{i}(t) \geq x_{i}\left(t^{\prime}\right) \exp \left(-\int_{t^{\prime}}^{t} \mu_{i}\left(w^{\top} x(s)\right) \mathrm{d} s\right)
$$

which is positive whenever $x_{i}\left(t^{\prime}\right)>0$. This establishes the desired inequality. 
In view of Lemma 8, we put

Definition 9 (Polymorphic trajectories). Any trajectory such that $u_{r}^{\top} x(0) \neq 0$ and $u_{s}^{\top} x(0) \neq 0$ is called a polymorphic trajectory.

Due to Lemma 8, any polymorphic trajectory is constituted of holomorphic states, except possibly at its initial point.

\subsection{Mean allelic mortality and recruitment rates}

In order to study selection in Section $[$, we associate here to each of the systems $(\mathbf{F})$ and $(\mathbf{S})$ two mean allelic rates, which are defined at any polymorphic state. Due to Lemma 8 and property 1 in Lemma 2 . these rates are well defined along any polymorphic trajectory.

Definition 10 (Mean allelic mortality and recruitment rates).

- System $(\underline{\mathbf{F}})$. For any polymorphic state $x \in \mathbb{R}_{+}^{3}$, define the mean allelic recruitment rates $\tilde{m}_{[\mathbf{F}}{ }_{j}(x)$ :

$$
\tilde{m}_{\mathbf{F}_{j}}(x):=\frac{u_{j}^{\top} M(x) x}{u_{j}^{\top} x}, \quad j=r, s
$$

and the mean allelic mortality rates $\tilde{\mu}_{\mathbf{F}}{ }_{j}(x)$ :

$$
\tilde{\mu} \mathbb{F}_{j}(x):=\frac{u_{j}^{\top} \mu\left(w^{\top} x\right) x}{u_{j}^{\top} x}, \quad j=r, s .
$$

- System ( $(\underline{\mathbf{S}})$. For any polymorphic state $x \in \mathbb{R}_{+}^{3}$, define the mean allelic recruitment rates $\tilde{m}\left[\mathbf{S}{ }_{j}(x)\right.$ :

$$
\tilde{m}_{\mathbf{S}_{j}}(x):=\frac{u_{j}^{\top} M(\alpha(x)) \alpha(x)}{u_{j}^{\top} x}, \quad j=r, s
$$

and the mean allelic mortality rates $\tilde{\mu} \mathbf{S}_{j}(x)$ :

$$
\tilde{\mu}\left[\mathbf{S}_{j}(x):=\frac{u_{j}^{\top} \mu\left(w^{\top} x\right) x}{u_{j}^{\top} x}, \quad j=r, s .\right.
$$

The fundamental interest of the previous definitions is to allow writing the evolution of the allelic populations along any polymorphic trajectory as:

$$
\begin{aligned}
u_{j}^{\top} \dot{x} & =u_{j}^{\top}\left(\alpha(M(x) x)-\mu\left(w^{\top} x\right) x\right)=u_{j}^{\top} M(x)-\tilde{\mu} \underline{\mathbf{F}}_{j}(x) u_{j}^{\top} x \\
& =\left(\tilde{m} \tilde{\mathbf{F}}_{j}(x)-\tilde{\mu} \mathbf{F}_{j}(x)\right) u_{j}^{\top} x, \quad j=r, s
\end{aligned}
$$

for system $(\underline{\mathbf{F}})$; and similarly for system $(\underline{\mathbf{S}})$ :

$$
\begin{aligned}
u_{j}^{\top} \dot{x} & =u_{j}^{\top}\left(M(\alpha(x)) \alpha(x)-\mu\left(w^{\top} x\right) x\right)=\left(\frac{u_{j}^{\top} M(\alpha(x)) \alpha(x)}{u_{j}^{\top} \alpha(x)}-\tilde{\mu} \overline{\mathbf{S}}_{j}(x)\right) u_{j}^{\top} x \\
& =\left(\tilde{m}[\mathbf{S}]_{j}(x)-\tilde{\mu}\left[\mathbf{S}_{j}(x)\right) u_{j}^{\top} x, \quad j=r, s .\right.
\end{aligned}
$$

(Property 1 of Lemma 2 was used in the previous deductions).

A key characteristic of the mean rates introduced in Definition 10 is summarized in the following lemma.

Lemma 11 (Ordering of the mean allelic rates). Assume Assumptions 1 and 2 are fulfilled. Then for any polymorphic state $x \in \mathbb{R}_{+}^{3}$, one has

$$
\begin{gathered}
m_{1}\left(b^{*}(x)\right) \geq \tilde{m}\left[\mathbf{F}{ }_{r}(x) \geq m_{2}\left(b^{*}(x)\right) \geq \tilde{m}_{[\mathbf{F}}(x) \geq m_{3}\left(b^{*}(x)\right),\right. \\
\left.m_{1}\left(b^{*}(\alpha(x))\right) \geq \tilde{m}_{[\mathbf{S}}(x) \geq m_{2}\left(b^{*}(\alpha(x))\right) \geq \tilde{m}_{[\mathbf{S}}\right]_{s}(x) \geq m_{3}\left(b^{*}(\alpha(x))\right)
\end{gathered}
$$

and

$$
\begin{gathered}
\mu_{1}\left(w^{\top} x\right) \leq \tilde{\mu} \overline{\mathbf{F}}_{r}(x) \leq \mu_{2}\left(w^{\top} x\right) \leq \tilde{\mu} \tilde{\mathbf{F}}_{s}(x) \leq \mu_{3}\left(w^{\top} x\right) \\
\mu_{1}\left(w^{\top} x\right) \leq \tilde{\mu} \mathbf{S}{ }_{r}(x) \leq \mu_{2}\left(w^{\top} x\right) \leq \tilde{\mu} \mathbf{S}_{s}(x) \leq \mu_{3}\left(w^{\top} x\right)
\end{gathered}
$$


Proof. For some $j \in\{r, s\}$ consider e.g. the map $\tilde{\mu} \mathbf{F} j$. One has

$$
\tilde{\mu}_{\mathbf{F}_{j}}(x):=\frac{u_{j}^{\top} \mu\left(w^{\top} x\right) x}{u_{j}^{\top} x}
$$

and thus

$$
\tilde{\mu}_{\mathbf{F}}{ }_{1}(x)=\frac{\mu_{1}\left(w^{\top} x\right) x_{1}+\frac{1}{2} \mu_{2}\left(w^{\top} x\right) x_{2}}{x_{1}+\frac{1}{2} x_{2}}, \quad \tilde{\mu}_{\mathbf{F}_{3}}(x)=\frac{\mu_{3}\left(w^{\top} x\right) x_{3}+\frac{1}{2} \mu_{2}\left(w^{\top} x\right) x_{2}}{x_{1}+\frac{1}{2} x_{2}}
$$

and Assumption 2 yields immediately (32a). The three other formulas are proved in the same way.

\section{Analysis of the selectively neutral case}

An inheritable variant is selectively neutral when the phenotypic manifestations of certain mutant alleles are equivalent to those of the wild type allele in their fitness (fertility and viability). Neutrality is considered the null hypothesis of evolution in the study of adaptation [48, since when this is not fulfilled, the selection operates.

We consider here the selectively neutral case, where recruitment and mortality rates are identical for all genotypes. Write in this case $\mu_{\mathrm{sn}}:=\mu_{i}, m_{\mathrm{sn}}:=m_{i}$ for $i=1,2,3$. In particular, Assumption 2 doesn't hold here. As a consequence of Assumption 1, $m_{\mathrm{sn}}$ is decreasing and $\mu_{\mathrm{sn}}$ increasing. Equation (18) here writes

$$
b=m_{\mathrm{sn}}(b) v^{\top} x
$$

and its unique scalar solution clearly depends upon $x$ only through the quantity $v^{\top} x$. To emphasize this fact, it is denoted $b_{\mathrm{sn}}\left(v^{\top} x\right)$, rather than $b^{*}(x)$. As a direct consequence of Lemma 1, $b_{\mathrm{sn}}$ is null at zero and takes on positive values otherwise. Also, it comes from the second point of Lemma 3 that $b_{\mathrm{sn}}$ is increasing. The following result for $(\underline{\mathbf{F}})$ and $(\underline{\mathbf{S}})$ then presents no difficulty.

Lemma 12. Assume Assumptions 1 , 3 and 4 are fulfilled. For any $x \in \mathbb{R}_{+}^{3} \backslash\{0\}$, there exists a unique solution, denoted $c_{\mathrm{sn}}^{*}(x)$ in the sequel, of the equation

$$
m_{\mathrm{sn}}\left(b_{\mathrm{sn}}\left(c v^{\top} x\right)\right)=\mu_{\mathrm{sn}}\left(c w^{\top} x\right) .
$$

The latter is such that the vector $c_{\mathrm{sn}}^{*}(x) x$ is an equilibrium.

Proof. Due to Assumption 1 the map $c \mapsto m_{\mathrm{sn}} \circ b_{\mathrm{sn}}\left(c v^{\top} x\right)$ is decreasing, while the map $c \mapsto \mu_{\mathrm{sn}}\left(c w^{\top} x\right)$ is increasing. On the other hand, $m_{\mathrm{sn}}\left(b_{\mathrm{sn}}(0)\right)=m_{\mathrm{sn}}(0)>\mu_{\mathrm{sn}}(0)$ from Assumption 3 and $0 \leq$ $\lim _{z \rightarrow+\infty} m_{\mathrm{sn}}(z)<\lim _{z \rightarrow+\infty} \mu_{\mathrm{sn}}(z) \leq+\infty$ due to Assumption 4. This demonstrates Lemma 12

Both systems $(\mathbf{F})$ and $(\underline{\mathbf{S}})$ now reduce to the equation

$$
\dot{x}=m_{\mathrm{sn}}\left(b_{\mathrm{sn}}\left(v^{\top} x\right)\right) \alpha(x)-\mu_{\mathrm{sn}}\left(w^{\top} x\right) x .
$$

The asymptotic behaviour of the solutions of (34) is completely described by the following fundamental result. In particular, its proof makes clear that the total population follows a variant of Verhulst's logistic growth equation [49].

Theorem 13 (Hardy-Weinberg law in selectively neutral evolution). Assume Assumptions 1, 3 and 9 are fulfilled, and that the recruitment and mortality rates are the same for all genotypes. Then for any nonzero initial condition, the solutions of $(\mathbf{F})$, resp. (S), satisfy

$$
\forall t \geq 0, \frac{u_{j}^{\top} x(t)}{\mathbf{1}^{\top} x(t)}=\frac{u_{j}^{\top} x(0)}{\mathbf{1}^{\top} x(0)}:=p_{j}, \quad j=r, s
$$

where $p_{r}+p_{s}=1$, and

$$
\lim _{t \rightarrow+\infty} x(t)=c_{\mathrm{sn}}^{*}(p) p
$$

for $c_{\mathrm{sn}}^{*}$ defined in Lemma 12 and the vector $p$ defined by $p^{\top}:=\left(\begin{array}{llll}p_{r}^{2} & 2 p_{r} p_{s} & p_{s}^{2}\end{array}\right)$. 
As is clear from the statement, Assumption 2, which implies fitness ordering, is not made here. Theorem 13 establishes that in absence of asymmetric fitness among genotypes, systems (F) and ( $(\mathbf{S})$ fulfil the Hardy-Weinberg principle: the allele frequencies remain constant over time and determine the asymptotic genotype frequencies, see (35), while the total density of individuals converges to the unique population level, defined by the solution of (33), which forms the carrying capacity for this relative frequency. This is a "null model" of the behavior of genes frequency in a population [50, obtained as a consequence of the random mating and the absence of selection in the population.

Proof of Theorem 13, - For any $j=r, s$, one has

$$
u_{j}^{\top} \dot{x}=u_{j}^{\top}\left(m_{\mathrm{sn}}\left(b_{\mathrm{sn}}\left(v^{\top} x\right)\right) \alpha(x)-\mu_{\mathrm{sn}}\left(w^{\top} x\right) x\right)=\left(m_{\mathrm{sn}}\left(b_{\mathrm{sn}}\left(v^{\top} x\right)\right)-\mu_{\mathrm{sn}}\left(w^{\top} x\right)\right) u_{j}^{\top} x
$$

using property 1 in Lemma 2 Therefore

$$
\mathbf{1}^{\top} \dot{x}=\left(u_{r}+u_{s}\right)^{\top} \dot{x}=\left(m_{\mathrm{sn}}\left(b_{\mathrm{sn}}\left(v^{\top} x\right)\right)-\mu_{\mathrm{sn}}\left(w^{\top} x\right)\right) \mathbf{1}^{\top} x
$$

The two ratios $\frac{u_{j}^{\top} x(t)}{\mathbf{1}^{\top} x(t)}$ are defined for any $t \geq 0$ and differentiable with respect to time, with

$$
\begin{aligned}
\frac{\mathrm{d}}{\mathrm{d} t}\left(\frac{u_{j}^{\top} x}{\mathbf{1}^{\top} x}\right) & =\frac{\left(u_{j}^{\top} \dot{x}\right)\left(\mathbf{1}^{\top} x\right)-\left(u_{j}^{\top} x\right)\left(\mathbf{1}^{\top} \dot{x}\right)}{\left(\mathbf{1}^{\top} x\right)^{2}} \\
& =\left(m_{\mathrm{sn}}\left(b_{\mathrm{sn}}\left(v^{\top} x\right)\right)-\mu_{\mathrm{sn}}\left(w^{\top} x\right)\right) \frac{\left(u_{j}^{\top} x\right)\left(\mathbf{1}^{\top} x\right)-\left(u_{j}^{\top} x\right)\left(\mathbf{1}^{\top} x\right)}{\left(\mathbf{1}^{\top} x\right)^{2}}=0
\end{aligned}
$$

which yields (35) by integration.

- One now studies the evolution of the components of the vector $x$. ite One may rewrite formally (34) as

$$
\dot{x}=\hat{m}(t) \alpha(x)-\hat{\mu}(t) x
$$

where for simplicity we put the scalar functions $\hat{m}(t):=m_{\mathrm{sn}}\left(b_{\mathrm{sn}}\left(v^{\top} x(t)\right)\right)$ and $\hat{\mu}(t):=\mu_{\mathrm{sn}}\left(w^{\top} x(t)\right)$. Using (35), which has just been demonstrated, one may write, for any $t \geq 0$,

$$
\alpha(x(t))=\frac{1}{\mathbf{1}^{\top} x(t)}\left(\begin{array}{c}
\left(u_{r}^{\top} x(t)\right)^{2} \\
2\left(u_{r}^{\top} x(t)\right)\left(u_{s}^{\top} x(t)\right) \\
\left(u_{s}^{\top} x(t)\right)^{2}
\end{array}\right)=\left(\mathbf{1}^{\top} x(t)\right) p
$$

for the vector $p$ defined in the statement. Consequently, one obtains from (37) the identities:

$$
\begin{aligned}
\forall t \geq 0, \hat{m}(t)\left(\mathbf{1}^{\top} x(t)\right) & =\frac{1}{p_{r}^{2}}\left(\dot{x}_{1}+\hat{\mu}(t) x_{1}(t)\right) \\
& =\frac{1}{2 p_{r} p_{s}}\left(\dot{x}_{2}+\hat{\mu}(t) x_{2}(t)\right) \\
& =\frac{1}{p_{s}^{2}}\left(\dot{x}_{3}+\hat{\mu}(t) x_{3}(t)\right)
\end{aligned}
$$

One then deduces that

$$
\frac{\mathrm{d}}{\mathrm{d} t}\left(\frac{x_{1}}{p_{r}^{2}}-\frac{x_{2}}{2 p_{r} p_{s}}\right)+\hat{\mu}(t)\left(\frac{x_{1}}{p_{r}^{2}}-\frac{x_{2}}{2 p_{r} p_{s}}\right)=\frac{\mathrm{d}}{\mathrm{d} t}\left(\frac{x_{3}}{p_{s}^{2}}-\frac{x_{2}}{2 p_{r} p_{s}}\right)+\hat{\mu}(t)\left(\frac{x_{3}}{p_{s}^{2}}-\frac{x_{2}}{2 p_{r} p_{s}}\right)=0 .
$$

The function $\hat{\mu}(t)$ is uniformly bounded from below by a positive constant on the set $\left\{x \in \mathbb{R}_{+}^{3}: \mathbf{1}^{\top} x \leq\right.$ $\left.c^{*}\right\}$, due to Theorem 4. Thus $\int_{0}^{+\infty} \hat{\mu}(t) d t=+\infty$, and one deduces by integration of (38) that the limits in the following formula exist, and consequently that the identities themselves are true:

$$
\lim _{t \rightarrow+\infty}\left(\frac{x_{1}(t)}{p_{r}^{2}}-\frac{x_{2}(t)}{2 p_{r} p_{s}}\right)=\lim _{t \rightarrow+\infty}\left(\frac{x_{3}(t)}{p_{s}^{2}}-\frac{x_{2}(t)}{2 p_{r} p_{s}}\right)=0 .
$$

Therefore, the evolution occurs asymptotically on the half-line

$$
\left\{x \in \mathbb{R}_{+}^{3}: \exists c>0, x=c p\right\} .
$$


- Now, the evolution of the state $x(t)=c(t) p$ on the previous half-line is dictated by the evolution of $c(t)$. The fact that $p_{r}+p_{s}=1$ implies that $\mathbf{1}^{\top} x(t)=c(t)$, and the function $c$ fulfils the scalar differential equation

$$
\dot{c}=(\hat{m}(t)-\hat{\mu}(t)) c(t)=\left(m_{\mathrm{sn}} \circ b_{\mathrm{sn}}\left(c(t) v^{\top} p\right)-\mu_{\mathrm{sn}}\left(c(t) w^{\top} p\right)\right) c(t) .
$$

The latter possesses two points of equilibrium, namely 0 and $c_{\mathrm{sn}}^{*}(p)$ by definition of the map $c_{\mathrm{sn}}^{*}$ - see Lemma 12 Due to Assumption 3 , one has $m_{\mathrm{sn}}(0)>\mu_{\mathrm{sn}}(0)$, so the first equilibrium is unstable, while the second one is globally asymptotically stable. This achieves the proof of Theorem 13

\section{Analysis of the selection case}

We now state the result that describes the asymptotic behaviour in the dominant and codominant cases.

Theorem 14 (Asymptotic convergence to the homozygous equilibrium with higher fitness). Assume

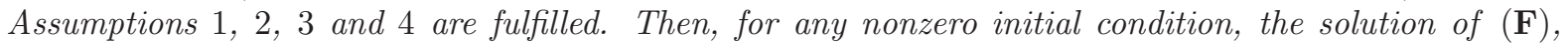
resp. (S), satisfies

$$
\lim _{t \rightarrow+\infty} x(t)=c_{1}^{*} e_{1}
$$

if allele $r$ is initially present, and otherwise

$$
\lim _{t \rightarrow+\infty} x(t)=c_{3}^{*} e_{3} .
$$

Theorem 14 states that, provided that the allele $r$ is initially present, the system converges asymptotically towards the homozygous equilibrium of strictly higher fitness. When only the allele $s$ is present, it goes towards the other homozygous nonzero equilibrium, or towards extinction if the latter does not exist. In both cases, the asymptotic population levels $c_{j}^{*}, j=r=1$ and $j=s=3$, correspond to the monomorphic equilibria defined in Lemma 6

Theorem 14 is a central result in the context of the evolution of resistance. It is important to emphasize that a scenario of resistance reversal would be covered by turning in the direction of the order relationship for the fitness components. From a biological point of view, the latter implies homogeneous modifications in the environment - in space and time interval - that condition heterogeneous fitness in a target population. This last Theorem guarantees that the selection can lead to total saturation, that is, to the fixing of the resistance and total adaptation of the population. Lemmas 17 and 18 guarantee the extinction of the susceptible allele. The latter is a fundamental difference in relation to the models derived from the conventional definition of Malthusian fitness, where all alleles grow exponentially unless they are assumed to be unviable (birth less than mortality), e.g. dynamics for Mendelian populations model presented in [17.

The case of monomorphic trajectories has been already studied in Lemma 7 . The proof of Theorem 14 in the general case of polymorphic trajectories is conducted in the remaining part of the present section, based on careful study of the evolution of each allele $j=r, s$ in the population, and then of the evolution of each genotypic population $i=1,2,3$. Central use will be made of the notions of mean allelic rates and of the formulas (30), introduced in Section 4.3, Most of the demonstration steps below are similar for $(\mathbf{E})$ and $(\underline{\mathbf{S}})$, and will be treated altogether for both systems. For simplicity we drop, whenever possible, the indices referring to the system considered, and simply write the allelic evolution

$$
u_{j}^{\top} \dot{x}=\left(\tilde{m}_{j}(x(t))-\tilde{\mu}_{j}(x(t))\right) u_{j}^{\top} x(t), \quad j=r, s .
$$

We first demonstrate in the following result that the ratio of the allelic frequencies evolves in a monotone way. More precisely, the ratio of the densities of the ("susceptible") allele $s$ over the ("resistant") allele $r$ is non increasing.

Lemma 15. For any polymorphic trajectory,

$$
\forall t \geq 0, \quad \frac{\mathrm{d}}{\mathrm{d} t}\left(\frac{u_{s}^{\top} x(t)}{u_{r}^{\top} x(t)}\right)=\left(\tilde{m}_{s}(x(t))-\tilde{\mu}_{s}(x(t))-\tilde{m}_{r}(x(t))+\tilde{\mu}_{r}(x(t))\right)\left(\frac{u_{s}^{\top} x(t)}{u_{r}^{\top} x(t)}\right) \leq 0,
$$

and the previous inequality is strict for any $t>0$. 
Proof.

- As a consequence of Lemma 8 the ratio $\frac{u_{s}^{\top} x(t)}{u_{r}^{\top} x(t)}$ is defined for any $t \geq 0$ along a polymorphic trajectory, and it is differentiable with respect to time. One has therefore

$$
\forall t \geq 0, \quad \frac{\mathrm{d}}{\mathrm{d} t}\left(\frac{u_{s}^{\top} x(t)}{u_{r}^{\top} x(t)}\right)=\left(\frac{u_{s}^{\top} \dot{x}}{u_{s}^{\top} x(t)}-\frac{u_{r}^{\top} \dot{x}}{u_{r}^{\top} x(t)}\right) \frac{u_{s}^{\top} x(t)}{u_{r}^{\top} x(t)}
$$

which, thanks to (41), gives the equality part of (42). Formulas (31) and (32) provide the non-strict version of (42).

- To prove the strict inequality, consider first system $(\mathbf{F})$. One has

$$
\begin{aligned}
& \left(\tilde{m} \mathbf{\mathbf { F }}{ }_{r}(x)-\tilde{\mu} \mathbf{F}{ }_{r}(x)-\tilde{m} \mathbf{F}_{s}(x)+\tilde{\mu} \mathbf{F} s(x)\right) \\
& =\left(\tilde{r}_{\mathbf{E}}{ }_{r}(x)-m_{2}\left(b^{*}(x)\right)-\tilde{\mu}_{\mathbf{F}} r(x)+\mu_{2}\left(w^{\top} x\right)\right) \\
& +\left(m_{2}\left(b^{*}(x)\right)-\tilde{m} \tilde{\mathbf{F}}_{s}(x)+\tilde{\mu}_{\mathbf{F}} s_{s}(x)-\mu_{2}\left(w^{\top} x\right)\right)
\end{aligned}
$$

and due to Lemma 11, each of the two expressions between parentheses in the right-hand side of (44) is nonnegative.

Moreover, at any polymorphic point, one has - see the definitions in (28):

$$
\begin{aligned}
& \tilde{m}_{\mathbf{F}}{ }_{r}(x)-m_{2}\left(b^{*}(x)\right)-\tilde{\mu} \mathbf{F}{ }_{r}(x)+\mu_{2}\left(w^{\top} x\right) \\
& =\frac{x_{1}}{u_{r}^{\top} x}\left(m_{1}\left(b^{*}(x)\right)-m_{2}\left(b^{*}(x)\right)+\mu_{2}\left(w^{\top} x\right)-\mu_{1}\left(w^{\top} x\right)\right) \\
& m_{2}\left(b^{*}(x)\right)-\tilde{m} \mathbf{F}{ }_{s}(x)+\tilde{\mu} \mathbf{F} s_{s}(x)-\mu_{2}\left(w^{\top} x\right) \\
& =\frac{x_{3}}{u_{s}^{\top} x}\left(m_{2}\left(b^{*}(x)\right)-m_{3}\left(b^{*}(x)\right)+\mu_{3}\left(w^{\top} x\right)-\mu_{2}\left(w^{\top} x\right)\right)
\end{aligned}
$$

A fundamental point now is that, due to the fact that $x_{1} \neq 0$ and $x_{3} \neq 0$ at any polymorphic point, one deduces from the second part of Assumption 2 that at least one of the two nonnegative expressions $m_{1}\left(b^{*}(x)\right)-m_{2}\left(b^{*}(x)\right)+\mu_{2}\left(w^{\top} x\right)-\mu_{1}\left(w^{\top} x\right)$ and $m_{2}\left(b^{*}(x)\right)-m_{3}\left(b^{*}(x)\right)+\mu_{3}\left(w^{\top} x\right)-\mu_{2}\left(w^{\top} x\right)$ is positive. Notice that Assumption 2 is crucial here for nonnegativity.

As all genotypes are present along a polymorphic trajectory when $t>0$ - see Lemma 8 one gets that, along any polymorphic trajectory, at least one of the two nonnegative expressions

$$
\frac{x_{1}(t)}{u_{r}^{\top} x(t)}\left(m_{1}\left(b^{*}(x(t))\right)-m_{2}\left(b^{*}(x(t))\right)+\mu_{2}\left(w^{\top} x(t)\right)-\mu_{1}\left(w^{\top} x(t)\right)\right)
$$

and

$$
\frac{x_{3}(t)}{u_{s}^{\top} x(t)}\left(m_{2}\left(b^{*}(x(t))\right)-m_{3}\left(b^{*}(x(t))\right)+\mu_{3}\left(w^{\top} x(t)\right)-\mu_{2}\left(w^{\top} x(t)\right)\right)
$$

is indeed positive whenever $t>0$. This in turn shows that along any polymorphic trajectory of equation $(\mathbf{F}),\left(\tilde{m}_{\mathbf{F}}(x(t))-\tilde{\mu}_{\mathbf{F}}(x(t))-\tilde{m} \tilde{\mathbf{F}}_{A}(x(t))+\tilde{\mu} \mathbf{F}{ }_{A}(x(t))\right)<0$ for any $t>0$, and thus the strict inequality in (42). This achieves the proof of Lemma 15 in the case of equation (F).

- The same argument holds for system (S). The counterpart of the formulas (45) is obtained by noticing that, at any polymorphic point, one has (see (29)):

$$
\begin{aligned}
& \tilde{m}_{\mathbf{S}_{r}}(x)-m_{2}\left(b^{*}(\alpha(x))\right)-\tilde{\mu} \mathbf{S}_{r}(x)+\mu_{2}\left(w^{\top} x\right) \\
& \quad=\frac{\alpha_{1}(x)}{\alpha_{1}(x)+\frac{1}{2} \alpha_{2}(x)}\left(m_{1}\left(b^{*}(\alpha(x))\right)-m_{2}\left(b^{*}(\alpha(x))\right)\right)+\frac{x_{1}}{u_{r}^{\top} x}\left(\mu_{2}\left(w^{\top} x\right)-\mu_{1}\left(w^{\top} x\right)\right) \\
& \quad=\frac{u_{r}^{\top} x}{\mathbf{1}^{\top} x}\left(m_{1}\left(b^{*}(\alpha(x))\right)-m_{2}\left(b^{*}(\alpha(x))\right)\right)+\frac{x_{1}}{u_{r}^{\top} x}\left(\mu_{2}\left(w^{\top} x\right)-\mu_{1}\left(w^{\top} x\right)\right)
\end{aligned}
$$

and similarly

$$
\begin{aligned}
\left.m_{2}\left(b^{*}(\alpha(x))\right)-\tilde{m}_{[\mathbf{S}}\right]_{s}(x)+ & \left.\tilde{\mu}_{\mathbf{S}}\right]_{s}(x)-\mu_{2}\left(w^{\top} x\right) \\
& =\frac{u_{s}^{\top} x}{\mathbf{1}^{\top} x}\left(m_{2}\left(b^{*}(\alpha(x))\right)-m_{3}\left(b^{*}(\alpha(x))\right)\right)+\frac{x_{3}}{u_{s}^{\top} x}\left(\mu_{3}\left(w^{\top} x\right)-\mu_{2}\left(w^{\top} x\right)\right) .
\end{aligned}
$$


Using the adequate version of the second part of Assumption 2 allows to obtain in the same manner than before the strict inequality in (42) for equation (S), and finally achieves the proof of Lemma 15.

We gather in the following result a series of estimates related to the genotypic frequencies.

Lemma 16. For any polymorphic trajectory, there exists $c_{1}>0$ such that

$$
u_{s}^{\top} x(t) \leq c_{1}\left(u_{r}^{\top} x(t)\right), \quad \forall t \geq 0 .
$$

Moreover, for any $\varepsilon>0$, there exist $c_{2}, c_{3} \geq 0$ such that $\forall t \geq \varepsilon$

$$
x_{2}(t) \leq c_{2} x_{1}(t), \quad x_{3}(t) \leq c_{3} x_{2}(t), \quad \frac{x_{1}(t)}{u_{r}^{\top} x(t)} \geq \frac{1}{1+\frac{1}{2} c_{2}}, \quad \frac{x_{3}(t)}{u_{s}^{\top} x(t)} \leq \frac{c_{3}}{c_{3}+\frac{1}{2}}
$$

Lemma 16 establishes that along any polymorphic trajectory, the ratio of the densities of susceptible over the density of resistants is bounded from above.

Proof.

- Formula (47) comes as direct consequence of Lemma 15] As a matter of fact, integrating (42) yields

$$
\left(\frac{u_{s}^{\top} x(t)}{u_{r}^{\top} x(t)}\right)=\exp \left(\int_{0}^{t}\left(\tilde{m}_{s}(x(t))-\tilde{\mu}_{r}(x(t))-\tilde{m}_{r}(x(t))+\tilde{\mu}_{r}(x(t))\right) d t\right)\left(\frac{u_{s}^{\top} x(0)}{u_{r}^{\top} x(0)}\right) \leq\left(\frac{u_{s}^{\top} x(0)}{u_{r}^{\top} x(0)}\right)
$$

for any $t \geq 0$, and therefore

$$
u_{s}^{\top} x(t) \leq \frac{u_{s}^{\top} x(0)}{u_{r}^{\top} x(0)}\left(u_{r}^{\top} x(t)\right):=c_{1}\left(u_{r}^{\top} x(t)\right), \quad \forall t \geq 0 .
$$

- Consider first system $\left(\underline{\mathbf{F}}\right.$. For any polymorphic trajectory one has $x_{1}(\varepsilon)>0$ for any $\varepsilon>0$, see the proof of Lemma 8. Within the present demonstration, one assumes for simplicity that $\varepsilon$ may be taken as zero, i.e. $x_{1}(0)>0$. Choose then positive constants $c_{2}, c_{3}$ such that

$$
c_{2}>\max \left\{\frac{x_{2}(0)}{x_{1}(0)} ; 2 c_{1}\right\}, \quad c_{3}<\max \left\{\frac{x_{2}(0)}{x_{3}(0)} ; \frac{2}{c_{1}}\right\}
$$

where $c_{1}$ is the positive constant in (47). One deduces successively from Assumption 2 and (47) that

$$
\begin{aligned}
u_{s}^{\top} M(x) x & =\frac{1}{2} m_{2}\left(b^{*}(x)\right) x_{2}+m_{3}\left(b^{*}(x)\right) x_{3} \\
& \leq \frac{1}{2} m_{2}\left(b^{*}(x)\right) x_{2}+m_{2}\left(b^{*}(x)\right) x_{3} \\
& =m_{2}\left(b^{*}(x)\right)\left(u_{s}^{\top} x\right) \\
& \leq c_{1} m_{2}\left(b^{*}(x)\right)\left(u_{r}^{\top} x\right) \\
& =c_{1} m_{2}\left(b^{*}(x)\right)\left(x_{1}+\frac{1}{2} x_{2}\right) \\
& \leq c_{1}\left(m_{1}\left(b^{*}(x)\right) x_{1}+\frac{1}{2} m_{2}\left(b^{*}(x)\right) x_{2}\right) \\
& =c_{1}\left(u_{r}^{\top} M(x) x\right)
\end{aligned}
$$

Using by turns (

$$
\begin{aligned}
\dot{x}_{2} & =\frac{2}{\mathbf{1}^{\top} M(x) x}\left(u_{r}^{\top} M(x) x\right)\left(u_{s}^{\top} M(x) x\right)-\mu_{2}\left(w^{\top} x\right) x_{2} \\
& \leq 2 c_{1} \frac{1}{\mathbf{1}^{\top} M(x) x}\left(u_{r}^{\top} M(x) x\right)^{2}-\mu_{2}\left(w^{\top} x\right) x_{2} \\
& \leq c_{2} \frac{1}{\mathbf{1}^{\top} M(x) x}\left(u_{r}^{\top} M(x) x\right)^{2}-\mu_{2}\left(w^{\top} x\right) x_{2} \\
& =c_{2}\left(\dot{x}_{1}+\mu_{1}\left(w^{\top} x\right) x_{1}\right)-\mu_{2}\left(w^{\top} x\right) x_{2}
\end{aligned}
$$


Therefore,

$$
\begin{aligned}
\dot{x}_{2}-c_{2} \dot{x}_{1} & \leq c_{2} \mu_{1}\left(w^{\top} x\right) x_{1}-\mu_{2}\left(w^{\top} x\right) x_{2} \\
& =-\mu_{1}\left(w^{\top} x\right)\left(x_{2}-c_{2} x_{1}\right)+\left(\mu_{1}\left(w^{\top} x\right)-\mu_{2}\left(w^{\top} x\right)\right) x_{2} \\
& \leq-\mu_{1}\left(w^{\top} x\right)\left(x_{2}-c_{2} x_{1}\right)
\end{aligned}
$$

where the last inequality has been deduced from the fact that $\mu_{2} \geq \mu_{1}$, see Assumption 2. Integrating inequality (52) and using (50) yields for any $t \geq 0$ :

$$
x_{2}(t)-c_{2} x_{1}(t) \leq \exp \left(-\int_{0}^{t} \mu_{1}\left(w^{\top} x(s)\right) \mathrm{d} s\right)\left(x_{2}(0)-c_{2} x_{1}(0)\right) \leq 0
$$

and the first part of (48).

On the other hand, one also deduces from ( $(\underline{\mathbf{F . b}}),(\underline{51}),(\mathbf{F . c})$ and the fact that $\mu_{3} \geq \mu_{2}$,

$$
\begin{aligned}
\dot{x}_{2} & =\frac{2}{\mathbf{1}^{\top} M(x) x}\left(u_{r}^{\top} M(x) x\right)\left(u_{s}^{\top} M(x) x\right)-\mu_{2}\left(w^{\top} x\right) x_{2} \\
& \geq \frac{2}{c_{1}} \frac{1}{\mathbf{1}^{\top} M(x) x}\left(u_{s}^{\top} M(x) x\right)^{2}-\mu_{2}\left(w^{\top} x\right) x_{2} \\
& \geq c_{3} \frac{1}{\mathbf{1}^{\top} M(x) x}\left(u_{s}^{\top} M(x) x\right)^{2}-\mu_{2}\left(w^{\top} x\right) x_{2} \\
& =c_{3}\left(\dot{x}_{3}+\mu_{3}\left(w^{\top} x\right) x_{3}\right)-\mu_{2}\left(w^{\top} x\right) x_{2} \\
& \geq c_{3} \dot{x}_{3}+\mu_{3}\left(w^{\top} x\right)\left(c_{3} x_{3}-x_{2}\right)
\end{aligned}
$$

Therefore

$$
\dot{x}_{2}-c_{3} \dot{x}_{3} \geq-\mu_{3}\left(w^{\top} x\right)\left(x_{2}-c_{3} x_{3}\right)
$$

which, taking into account the fact that $c_{3}<\frac{x_{2}(0)}{x_{3}(0)}$, yields

$$
\forall t \geq 0, \quad x_{2}(t)-c_{3} x_{3}(t) \geq 0 .
$$

This proves the second inequality in (48). The last two inequalities come as a consequence, using the fact that

$$
\frac{x_{1}(t)}{u_{r}^{\top} x(t)}=\frac{x_{1}(t)}{x_{1}(t)+\frac{1}{2} x_{2}(t)}, \quad \frac{x_{3}(t)}{u_{s}^{\top} x(t)}=\frac{x_{3}(t)}{x_{3}(t)+\frac{1}{2} x_{2}(t)} .
$$

This concludes the proof of Lemma 16 in the case of system (F).

- The proof is conducted similarly for system ( $(\underline{\mathbf{S}})$. We just quote here step by step the differences in the computations. The analogue of formulas (52) and (53) is proved as follows. Using (S.b), Assumption 2 (47) and (S.a), one deduces that

$$
\begin{aligned}
\dot{x}_{2} & =\frac{2}{\mathbf{1}^{\top} x}\left(u_{r}^{\top} x\right)\left(u_{s}^{\top} x\right) m_{2}\left(b^{*}(\alpha(x))\right)-\mu_{2}\left(w^{\top} x\right) x_{2} \\
& \leq 2 c_{1} \frac{\left(u_{r}^{\top} x\right)^{2}}{\mathbf{1}^{\top} x} m_{1}\left(b^{*}(\alpha(x))\right)-\mu_{2}\left(w^{\top} x\right) x_{2} \\
& \leq c_{2} \frac{\left(u_{r}^{\top} x\right)^{2}}{\mathbf{1}^{\top} x} m_{1}\left(b^{*}(\alpha(x))\right)-\mu_{2}\left(w^{\top} x\right) x_{2} \\
& =c_{2}\left(\dot{x}_{1}+\mu_{1}\left(w^{\top} x\right) x_{1}\right)-\mu_{2}\left(w^{\top} x\right) x_{2}
\end{aligned}
$$

and therefore

$$
\begin{aligned}
\dot{x}_{2}-c_{2} \dot{x}_{1} & \leq c_{2} \mu_{1}\left(w^{\top} x\right) x_{1}-\mu_{2}\left(w^{\top} x\right) x_{2} \\
& =-\mu_{1}\left(w^{\top} x\right)\left(x_{2}-c_{2} x_{1}\right)+\left(\mu_{1}\left(w^{\top} x\right)-\mu_{2}\left(w^{\top} x\right)\right) x_{2} \\
& \leq-\mu_{1}\left(w^{\top} x\right)\left(x_{2}-c_{2} x_{1}\right)
\end{aligned}
$$


On the other hand, the fact that $m_{3} \leq m_{2}$ and $\mu_{3} \geq \mu_{2}$ yields

$$
\begin{aligned}
\dot{x}_{2} & =\frac{2}{\mathbf{1}^{\top} x}\left(u_{r}^{\top} x\right)\left(u_{s}^{\top} x\right) m_{2}\left(b^{*}(\alpha(x))\right)-\mu_{2}\left(w^{\top} x\right) x_{2} \\
& \geq \frac{2}{c_{1}} \frac{\left(u_{s}^{\top} x\right)^{2}}{\mathbf{1}^{\top} x} m_{3}\left(b^{*}(\alpha(x))\right)-\mu_{2}\left(w^{\top} x\right) x_{2} \\
& \geq \frac{1}{c_{3}} \frac{\left(u_{s}^{\top} x\right)^{2}}{\mathbf{1}^{\top} x} m_{3}\left(b^{*}(\alpha(x))\right)-\mu_{2}\left(w^{\top} x\right) x_{2} \\
& =\frac{1}{c_{3}}\left(\dot{x}_{3}+\mu_{3}\left(w^{\top} x\right) x_{3}\right)-\mu_{2}\left(w^{\top} x\right) x_{2} \\
& \geq \frac{1}{c_{3}} \dot{x}_{3}+\mu_{3}\left(w^{\top} x\right)\left(\frac{1}{c_{3}} x_{3}-x_{2}\right)
\end{aligned}
$$

which is the counterpart of (53). The other steps of the proof are similar to the case of system (E), and not repeated for the sake of space. This concludes the proof of Lemma 16.

We are now in position to show that the ratio of the allelic frequencies not only decreases, as demonstrated by the previous result, but also vanishes asymptotically. Of course Assumption 2, which orders the different recruitment and mortality rates, is crucial to this Lemma.

Lemma 17. For any polymorphic trajectory,

$$
\lim _{t \rightarrow+\infty} \frac{u_{s}^{\top} x(t)}{u_{r}^{\top} x(t)}=0
$$

Proof. We consider in the whole demonstration a given polymorphic trajectory - either of system (F) or of system $(\underline{\mathbf{S}})$ according to the context.

The ratio of allelic frequencies being decreasing, as a consequence of Lemma 15, there exists a nonnegative scalar $\lambda$ such that

$$
\lim _{t \rightarrow+\infty} \frac{u_{s}^{\top} x(t)}{u_{r}^{\top} x(t)}=\lambda
$$

Due to the fact that $u_{s}^{\top} x+u_{r}^{\top} x=\mathbf{1}^{\top} x$, one may deduce from (55) that the allelic frequencies also converge, namely:

$$
\lim _{t \rightarrow+\infty} \frac{u_{s}^{\top} x(t)}{\mathbf{1}^{\top} x(t)}=\frac{\lambda}{1+\lambda}, \quad \lim _{t \rightarrow+\infty} \frac{u_{r}^{\top} x(t)}{\mathbf{1}^{\top} x(t)}=\frac{1}{1+\lambda} .
$$

We assume by contradiction that

$$
\lambda>0 \text {. }
$$

Our aim is to show that (56) is wrong, i.e. that $\lambda=0$.

- By Theorem 4, the trajectories are uniformly bounded. Therefore, by compactness, Assumption 2 guarantees the existence of a certain $\zeta>0$ such that

$$
\forall t>0, \quad m_{1}\left(b^{*}(x(t))\right)-m_{3}\left(b^{*}(x(t))\right)+\mu_{3}\left(w^{\top} x(t)\right)-\mu_{1}\left(w^{\top} x(t)\right)>\zeta
$$

for system ( $(\underline{\mathbf{F}})$; and

$$
\forall t>0, \quad m_{1}\left(b^{*}(\alpha(x(t)))\right)-m_{3}\left(b^{*}(\alpha(x(t)))\right)+\mu_{3}\left(w^{\top} x(t)\right)-\mu_{1}\left(w^{\top} x(t)\right)>\zeta
$$

for system $(\underline{\mathbf{S}})$. For the considered trajectory and the corresponding value $\zeta$, let the set $X$ be defined as

$$
X:=\left\{x \in \mathbb{R}_{+}^{3}: m_{1}\left(b^{*}(x)\right)-m_{2}\left(b^{*}(x)\right)+\mu_{2}\left(w^{\top} x\right)-\mu_{1}\left(w^{\top} x\right)>\frac{\zeta}{2}\right\}
$$

for the case of system ( $[\mathbf{F}$, and as

$$
X:=\left\{x \in \mathbb{R}_{+}^{3}: m_{1}\left(b^{*}(\alpha(x))\right)-m_{2}\left(b^{*}(\alpha(x))\right)+\mu_{2}\left(w^{\top} x\right)-\mu_{1}\left(w^{\top} x\right)>\frac{\zeta}{2}\right\}
$$


for system $(\underline{\mathbf{S}})$. Notice that, due to (57),

$$
x(t) \notin X \Rightarrow m_{2}\left(b^{*}(x(t))\right)-m_{3}\left(b^{*}(x(t))\right)+\mu_{3}\left(w^{\top} x(t)\right)-\mu_{2}\left(w^{\top} x(t)\right)>\frac{\zeta}{2}
$$

for $(\mathbf{F})$, and for $(\underline{\mathbf{S}})$ :

$$
x(t) \notin X \Rightarrow m_{2}\left(b^{*}(\alpha(x(t)))\right)-m_{3}\left(b^{*}(\alpha(x(t)))\right)+\mu_{3}\left(w^{\top} x(t)\right)-\mu_{2}\left(w^{\top} x(t)\right)>\frac{\zeta}{2} .
$$

Now, observe that the derivative of $\frac{u_{s}^{\top} x}{u_{r}^{\top} x}$ appears in (42) as a locally Lipschitz function of the state variable $x$; and that along any polymorphic trajectory, the latter is uniformly bounded with uniformly bounded time derivative. From this, we may deduce that this derivative is uniformly continuous with respect to the time variable. As the convergence property (55) holds, Barbalat's lemma [51, 52, establishes that the derivative converges to zero when $t \rightarrow+\infty$. Inserting in the expression of the latter (see (42)), the decomposition in two nonnegative terms obtained in (44) and (45) for (F), and in (46) for (S), one concludes by use of the hypothesis (56), that

$$
\begin{aligned}
& \lim _{t \rightarrow+\infty} \frac{x_{1}(t)}{u_{r}^{\top} x(t)}\left(m_{1}\left(b^{*}(x(t))\right)-m_{2}\left(b^{*}(x(t))\right)+\mu_{2}\left(w^{\top} x(t)\right)-\mu_{1}\left(w^{\top} x(t)\right)\right)=0 \\
& \lim _{t \rightarrow+\infty} \frac{x_{3}(t)}{u_{s}^{\top} x(t)}\left(m_{2}\left(b^{*}(x(t))\right)-m_{3}\left(b^{*}(x(t))\right)+\mu_{3}\left(w^{\top} x(t)\right)-\mu_{2}\left(w^{\top} x(t)\right)\right)=0
\end{aligned}
$$

- Assume first that, for the considered polymorphic trajectory and the set $X$ defined in (58),

$$
\text { meas }\{t>0: x(t) \in X\}=+\infty,
$$

where meas denotes the Lebesgue measure.

Consider primarily the case of system (F). One derives from (45), (48) and the definition of $X$ in (58), that

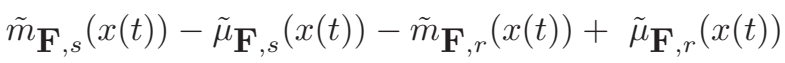

$$
\begin{aligned}
& \leq-\frac{x_{1}(t)}{u_{r}^{\top} x(t)}\left(m_{1}\left(b^{*}(x(t))\right)-m_{2}\left(b^{*}(x(t))\right)+\mu_{2}\left(w^{\top} x(t)\right)-\mu_{1}\left(w^{\top} x(t)\right)\right) \\
& \leq-\frac{1}{1+\frac{1}{2} c_{2}} \frac{\zeta}{2} \chi_{x^{-1}(X)}(t)
\end{aligned}
$$

where by definition the characteristic function $\chi_{x^{-1}(X)}(t)$ is equal to 1 if $x(t) \in X, 0$ otherwise. Integrating now this inequality as in (49), one gets for any $t \geq 0$,

$$
\left(\frac{u_{s}^{\top} x(t)}{u_{r}^{\top} x(t)}\right) \leq\left(\frac{u_{s}^{\top} x(0)}{u_{r}^{\top} x(0)}\right) \exp \left(-\frac{1}{1+\frac{1}{2} c_{2}} \frac{\zeta}{2} \operatorname{meas}\{s \in(0, t): x(s) \in X\}\right)
$$

Due to the hypothesis made in (61), the previous expression converges towards 0 when $t \rightarrow+\infty$. Therefore $\lambda=\lim _{t \rightarrow+\infty} \frac{u_{s}^{\top} x(t)}{u_{r}^{\top} x(t)}=0$, which contradicts (56). This latter formula is thus wrong, which establishes (54) by contradiction.

The case of (표 is quite similar. Due to (47), one has for any $t \geq 0$,

$$
\frac{u_{r}^{\top} x(t)}{\mathbf{1}^{\top} x(t)} \geq \frac{1}{1+c_{1}} \quad \text { and } \quad \frac{u_{s}^{\top} x(t)}{\mathbf{1}^{\top} x(t)} \leq \frac{c_{1}}{1+c_{1}} .
$$

From 46a), one derives here

$$
\begin{aligned}
& \left.\tilde{m}_{[\mathbf{S}}\right]_{s}(x(t))-\tilde{\mu} \mathbf{S}_{s}(x(t))-\tilde{m} \tilde{\mathbf{S}}_{r}(x(t))+\tilde{\mu} \mathbf{S}_{r}(x(t)) \\
& \leq-\frac{u_{r}^{\top} x}{\mathbf{1}^{\top} x}\left(m_{1}\left(b^{*}(\alpha(x))\right)-m_{2}\left(b^{*}(\alpha(x))\right)\right)-\frac{x_{1}}{u_{r}^{\top} x}\left(\mu_{2}\left(w^{\top} x\right)-\mu_{1}\left(w^{\top} x\right)\right) \\
& \leq-\frac{1}{1+c_{1}}\left(m_{1}\left(b^{*}(\alpha(x))\right)-m_{2}\left(b^{*}(\alpha(x))\right)\right)-\frac{1}{1+\frac{1}{2} c_{2}}\left(\mu_{2}\left(w^{\top} x\right)-\mu_{1}\left(w^{\top} x\right)\right) \\
& \leq-\min \left\{\frac{1}{1+c_{1}} ; \frac{1}{1+\frac{1}{2} c_{2}}\right\} \frac{\zeta}{2} \chi_{x^{-1}(X)}(t)
\end{aligned}
$$


which is analogous to (62). The demonstration is then conducted in the same way and yields similarly $\lambda=0$. By contradiction with (56), this shows identity (54).

- We now treat the case where (61) does not hold, that is:

$$
\text { meas }\{t>0: x(t) \in X\}<+\infty \text {. }
$$

It is not possible to use here the same argument than previously, because the quantity $\frac{x_{3}(t)}{u_{s}^{\top} x(t)}$ is bounded from above, contrary to $\frac{x_{1}(t)}{u_{r}^{\top} x(t)}$ which is bounded from below, see (48). The measure of the set $\{t>0: x(t) \notin X\}$ is now infinite, and as a consequence of (59), one deduces that

$$
\text { meas }\left\{t \geq 0: m_{2}\left(b^{*}(x(t))\right)-m_{3}\left(b^{*}(x(t))\right)+\mu_{3}\left(w^{\top} x(t)\right)-\mu_{2}\left(w^{\top} x(t)\right)>\frac{\zeta}{2}\right\}=+\infty
$$

for $(\underline{\mathbf{F}})$, and for $(\underline{\mathbf{S}})$ :

$$
\text { meas }\left\{t \geq 0: m_{2}\left(b^{*}(\alpha(x(t)))\right)-m_{3}\left(b^{*}(\alpha(x(t)))\right)+\mu_{3}\left(w^{\top} x(t)\right)-\mu_{2}\left(w^{\top} x(t)\right)>\frac{\zeta}{2}\right\}=+\infty .
$$

From (60) and (64), one obtains here that necessarily:

$$
\lim _{t \rightarrow+\infty} \frac{x_{3}(t)}{u_{s}^{\top} x(t)}=0
$$

and thus

$$
\lim _{t \rightarrow+\infty} x_{3}(t)=0 \text {. }
$$

In view of the equations $(\underline{\mathbf{F . c}})$ for system $(\underline{\mathbf{F}})$ and $(\underline{\text { S.c }})$ for system $(\underline{\mathbf{S}})$, one deduces by invoking newly Barbalat's lemma that

$$
\lim _{t \rightarrow+\infty} \dot{x}_{3}(t)=0 \text {. }
$$

By considering again the right-hand side of ( $(\underline{\mathbf{F . c}})$, resp. (…c), one then gets from (65) and (66) that

$$
\lim _{t \rightarrow+\infty} u_{s}^{\top} M(x(t)) x(t)=0, \quad \text { resp. } \quad \lim _{t \rightarrow+\infty} u_{s}^{\top} x(t)=0
$$

and therefore

$$
\lim _{t \rightarrow+\infty} x_{2}(t)=0
$$

But (65) and (67) together yield

$$
\lambda=\lim _{t \rightarrow+\infty} \frac{u_{s}^{\top} x(t)}{\mathbf{1}^{\top} x(t)}=0
$$

which contradicts (56). The latter is therefore wrong. This establishes (54) in the case where (63) holds, and finally concludes the proof of Lemma 17

As a remark, notice that using the techniques in the proof of Lemma 17, one may show that the convergence is exponential in (54) whenever the following stronger form of Assumption 2 holds: $m_{1}\left(b^{*}(x)\right)-m_{2}\left(b^{*}(x)\right)+\mu_{2}\left(w^{\top} x\right)-\mu_{1}\left(w^{\top} x\right)>0$ for system $(\mathbf{F})$, or $m_{1}\left(b^{*}(\alpha(x))\right)-m_{2}\left(b^{*}(\alpha(x))\right)+$ $\mu_{2}\left(w^{\top} x\right)-\mu_{1}\left(w^{\top} x\right)>0$ for system (S- Indeed (61) always holds in such cases. On the contrary, there is no indication that the same property holds when $m_{2}\left(b^{*}(x)\right)-m_{3}\left(b^{*}(x)\right)+\mu_{3}\left(w^{\top} x\right)-\mu_{2}\left(w^{\top} x\right)>0$ for system ( $(\mathbf{F})$, or $m_{2}\left(b^{*}(\alpha(x))\right)-m_{3}\left(b^{*}(\alpha(x))\right)+\mu_{3}\left(w^{\top} x\right)-\mu_{2}\left(w^{\top} x\right)>0$ for system (S).

The asymptotic behavior of the ratio of allelic populations given in Lemma 17 is sufficient to assert the asymptotics of the genotypic relative frequencies in $(\mathbf{F})$ and $(\underline{\mathbf{S}})$, as shown now.

Lemma 18. For any polymorphic trajectory,

$$
\lim _{t \rightarrow+\infty} \frac{x_{1}(t)}{\mathbf{1}^{\top} x(t)}=1, \quad \lim _{t \rightarrow+\infty} \frac{x_{2}(t)}{\mathbf{1}^{\top} x(t)}=\lim _{t \rightarrow+\infty} \frac{x_{3}(t)}{\mathbf{1}^{\top} x(t)}=0 .
$$


Proof. Recall that by definition

$$
\frac{u_{s}^{\top} x(t)}{u_{r}^{\top} x(t)}=\frac{\frac{1}{2} x_{2}(t)+x_{3}(t)}{x_{1}(t)+\frac{1}{2} x_{2}(t)} \geq \frac{\frac{1}{2} x_{2}(t)+x_{3}(t)}{x_{1}(t)+x_{2}(t)+x_{3}(t)}
$$

which is larger than or equal to both nonnegative expressions

$$
\frac{1}{2} \frac{x_{2}(t)}{x_{1}(t)+x_{2}(t)+x_{3}(t)} \quad \text { and } \quad \frac{x_{3}(t)}{x_{1}(t)+x_{2}(t)+x_{3}(t)}
$$

Lemma 17 implies that both these ratios converge towards 0 when $t \rightarrow+\infty$ and this permits to conclude the proof of Lemma 18

Due to Lemma 18 and the fact that the trajectories are bounded (see Theorem 41), $x_{2}$ and $x_{3}$ converge towards 0 . We are finally in the position to establish the asymptotic behaviour of the population size for each genotype.

Lemma 19. For any polymorphic trajectory,

$$
\lim _{t \rightarrow+\infty} x_{1}(t)=c_{1}^{*}, \quad \lim _{t \rightarrow+\infty} x_{2}(t)=\lim _{t \rightarrow+\infty} x_{3}(t)=0 .
$$

Proof. Consider e.g. system (F). Equation (F.a may be written as

$$
\dot{x}_{1}=\frac{\left(u_{r}^{\top} M(x) x\right)^{2}}{\mathbf{1}^{\top} M(x) x}-\mu_{1}\left(w^{\top} x\right) x_{1}=\left(\frac{\left(u_{r}^{\top} M(x) x\right)^{2}}{\left(\mathbf{1}^{\top} M(x) x\right) x_{1}}-\mu_{1}\left(w^{\top} x\right)\right) x_{1} .
$$

Due to (68),

$$
\lim _{t \rightarrow+\infty} \eta(t)=0, \quad \eta(t):=\left(\frac{\left(u_{r}^{\top} M(x) x\right)^{2}}{\left(\mathbf{1}^{\top} M(x) x\right) x_{1}}-\mu_{1}\left(w^{\top} x\right)-m_{1}\left(b^{*}\left(x_{1}(t) e_{1}\right)\right)+\mu_{1}\left(w_{1} x_{1}(t)\right)\right) .
$$

Thus, for any polymorphic trajectory of $(\mathbf{E})$ and any $\bar{\eta}>0$, there exists $T_{\bar{\eta}}>0$ such that, for any $t \geq T_{\bar{\eta}}$,

$$
\left(m_{1}\left(b^{*}\left(x_{1}(t) e_{1}\right)\right)-\mu_{1}\left(w_{1} x_{1}(t)\right)-\bar{\eta}\right) x_{1} \leq \dot{x}_{1} \leq\left(m_{1}\left(b^{*}\left(x_{1}(t) e_{1}\right)\right)-\mu_{1}\left(w_{1} x_{1}(t)\right)+\bar{\eta}\right) x_{1} .
$$

For $\bar{\eta}>0$ sufficiently small, let $c_{\bar{\eta}}^{ \pm}$be the unique positive scalars such that

$$
m_{1}\left(b^{*}\left(c_{\bar{\eta}}^{ \pm} e_{1}\right)\right)-\mu_{1}\left(w_{1} c_{\bar{\eta}}^{ \pm}\right) \pm \bar{\eta}=0 .
$$

By definition of $c_{1}^{*}$, see Lemma 6 , one has

$$
c_{\bar{\eta}}^{-}<c_{1}^{*}<c_{\bar{\eta}}^{+}, \quad \lim _{\bar{\eta} \rightarrow 0^{+}} c_{\overline{\bar{\eta}}}^{ \pm}=c_{1}^{*} .
$$

By (70), one deduces that, for any sufficiently small $\bar{\eta}>0$,

$$
c_{\bar{\eta}}^{-} \leq \liminf _{t \rightarrow+\infty} x_{1}(t) \leq \limsup _{t \rightarrow+\infty} x_{1}(t) \leq c_{\bar{\eta}}^{+},
$$

and finally

$$
\liminf _{t \rightarrow+\infty} x_{1}(t)=\limsup _{t \rightarrow+\infty} x_{1}(t) \leq c_{1}^{*},
$$

by doing $\bar{\eta} \rightarrow 0^{+}$and using (71). This demonstrates Lemma 19 in the case of system (F). System (S) is treated analogously.

With the proof of Lemma 19 the proof of Theorem 14 is now complete. 


\section{Conclusion and future issues}

We proposed a class of Mendelian inheritance models that considers the complexity of the life history of insects and its selective pressures. These models describe the evolution in continuous time of a population with two main life phases, governed by birth of the three genotypes of two alleles, density-dependent mortality rates and constant rate of passage to reproductive phase. Each model is represented by a system of six scalar ordinary differential equations, one for each genotype in each life phase.

This starting system has been simplified using slow manifold theory, to obtain two classes of Mendelian inheritance models of dimension 3. Both classes were derived from assuming one of the phases slower than the other.

We proved that, under realistic assumptions, the proposed models demonstrate several fundamental properties expected in population dynamics and population genetics. In fact, in a selectively neutral scenario, the population converges asymptotically to a carrying capacity, while Hardy-Weinberg law is valid: the allele frequencies in the polymorphic population are constant, and determine the asymptotic value of the genotypic relative frequencies. In presence of selective pressures -i.e. an ordering of the recruitment or mortality functions according to genotype-, adaptive evolution occurs, and in case of dominance or codominance, the population is asymptotically made of individuals of the homozygous genotype having the highest fitness, at the corresponding carrying capacity.

The evolution described above take place as consequence of a stationary environment. On the other hand, introducing time-dependence in the model parameters offers the possibility to describe a variety of evolving phenomena, such as e.g. emergence and reversal of resistance evolution as a result of intermittent pesticide applications. Also, these models allow to analyze selective pressure situations faced by genotypes during life phases occurring in different ecological niches.

The proposed classes of models permit, possibly through minor extensions, to study several issues of importance related to the use of insecticides and other adaptive phenomena. Simple extensions of the models to incorporate migration would allow to evaluate the consequences of the natural or artificial addition of susceptible genotypes in the evolution of insecticide resistance, following the proposals made in [28] and 29. Also, one could explore the consequences of the use of a larvicide and adulticide in the context of an evolution-proof insecticide following the line developed in [34, 12, 35], - partitioning the adult phase into younger adults and less vigorous old adults, with a mortality rate in the last phase more affected by the adulticide. Further, the proposed models can be used to explore how dominance levels given by the dose of insecticide [53, 54, 29, 55] affect the speed of settlement of resistance evolution [56, 50, 57. In addition, in situations where the application of the insecticide is interrupted and the resistance has a fitness cost, the models may be used to estimate the time taken by a resistant population to revert to a susceptible one, as done in [58, 37.

Looking forward, as most traits of evolutionary or economic importance are determined by several genes, an adequate understanding of the evolution of such traits may require the study of multi-locus models 21. It turns out that the heredity function which models the births is constructed in such a way as to allow extensions to more than two alleles, multiple loci - by means of Kronecker product between inheritance matrices - or polyploid cases. The modelling procedure presented in the present paper thus offers the ability to accommodate more complicated inheritance configurations, with two or more loci with autosomal inheritance, accounting for e.g. sequential or mixed use of insecticides 31, 32, 26, 33, nongenetic inheritance, unified maternal and autosomal inheritance as autosomal resistance and Wolbachia for biological control - a bacterium of maternal inheritance related to mitochondria with the potential to suppress vector capacity in mosquitoes [59, 14].

As a last remark, notice that models of species having more than two main phases may be considered too, for example in the case of a holometabolous insect for which the phases of embryo, larva and pupa are all quite fast compared to the adult phase. Note also that organism models having two life phases do not concern only insects. Even in plants it is possible to describe pre-reproductive and reproductive phases with different periods. For instance, weedy ephemerals (a very common weed) are very shortlived plants that reproduce rapidly after human disturbance from plowing 60. This means that the models proposed here could cover problems related to resistance to herbicides, where chemical control can occur in the seedling phase or in the reproductive phase (see 36]), which are supposed to slow down or even reverse the development of resistances due to the interruption or modification of the application of pesticide lethal doses in the environment.

Mathematical models played a decisive role in reconciling Mendelian genetics with Darwin's theory of 
adaptive evolution [18]. In regard to inheritance models, the biological or genetic control for insect pest raises new issues with potentially valuable applications 61, 62. We think that the modeling strategy presented here may help in this task, for which extensions of these models may help to design, analyze, dimension and simulate release strategies.

\section{Acknowledgments}

P.E.P.E. acknowledges the support of CONACyT in the framework of FEEI-PROCIENCIA program (POS007, POSG17-53, PVCT15-273, PVCT17-156, PVCT18-53). C.E.S. and P.E.P.E. acknowledge the CONACyT-PRONII program. All authors acknowledge the support of the STIC AmSud projects MOSTICAW (2016-2017) and NEMBICA (2020-2021).

\section{A Proof of technical results}

\section{A.1 Proof of Lemma 1}

The left-hand side of (18) is an increasing function of $b$ and varies from 0 to $+\infty$; while the right-hand side is null if $x=0_{3}$ and a decreasing function otherwise. Therefore, by the implicit function theorem, there exists a unique solution $b^{*}(x)$ to the scalar equation

$$
b-\sum_{i=1}^{3} v_{i} m_{i}(b) x_{i}=0
$$

which is of class $C^{p}$ if every function $m_{i}$ is of class $C^{p}, p \in \mathbb{N}$.

\section{A.2 Proof of Lemma 2}

For Property 1 one can see that, for any $j=r, s$,

$$
u_{j}^{\top} \alpha(x)=\frac{1}{\mathbf{1}^{\top} x}\left(\left(u_{j}^{\top} x\right)^{2}+\left(u_{r}^{\top} x\right)\left(u_{s}^{\top} x\right)\right)=\frac{1}{\mathbf{1}^{\top} x}\left(u_{r}^{\top} x+u_{s}^{\top} x\right) u_{j}^{\top} x=u_{j}^{\top} x .
$$

as $u_{r}+u_{s}=\mathbf{1}$. Using again this identify, property 2 is deduced from the previous one, as:

$$
\mathbf{1}^{\top} \alpha(x)=u_{r}^{\top} \alpha(x)+u_{s}^{\top} \alpha(x)=u_{r}^{\top} x+u_{s}^{\top} x=\mathbf{1}^{\top} x .
$$

Property 3 is trivial, and expresses the homogeneity of the function $\alpha$. To show Property 4 . notice that for any $(j, k) \in\{1,3\} \times\{r, s\}, u_{k}^{\top} e_{j}=1$ if $(j, k)=(1, r)$ or $(3, s)$, and 0 otherwise; and on the other hand that $\mathbf{1}^{\top} e_{j}=1$. Therefore

$$
\alpha\left(e_{j}\right)=\frac{1}{\mathbf{1}^{\top} e_{j}}\left(\begin{array}{c}
\left(u_{r}^{\top} e_{j}\right)^{2} \\
2\left(u_{r}^{\top} e_{j}\right)\left(u_{s}^{\top} e_{j}\right) \\
\left(u_{s}^{\top} e_{j}\right)^{2}
\end{array}\right)=e_{j}
$$

for any $j=r=1, j=s=3$.

\section{A.3 Proof of Lemma 3}

- Let $x \in \mathbb{R}_{+}^{3} \backslash\left\{0_{3}\right\}$ and $\lambda>0$. By definition (see the statement of Lemma 1),

$$
b^{*}(\lambda x)=\lambda \sum_{i=1}^{3} v_{i} m_{i}\left(b^{*}(\lambda x)\right) x_{i} .
$$

Therefore

$$
\lambda \min _{i=1,2,3}\left\{v_{i} / w_{i}\right\} \min _{i=1,2,3}\left\{m_{i}\left(b^{*}(\lambda x)\right)\right\} w^{\top} x \leq b^{*}(\lambda x) \leq \lambda \max _{i=1,2,3}\left\{v_{i} / w_{i}\right\} \max _{i=1,2,3}\left\{m_{i}\left(b^{*}(\lambda x)\right)\right\} w^{\top} x .
$$


which may be rewritten as an inequality on $\lambda\left(w^{\top} x\right)$ :

$$
\frac{b^{*}(\lambda x)}{\max _{i}\left\{v_{i} / w_{i}\right\} \max _{i}\left\{m_{i}\left(b^{*}(\lambda x)\right)\right\}} \leq \lambda\left(w^{\top} x\right) \leq \frac{b^{*}(\lambda x)}{\min _{i}\left\{v_{i} / w_{i}\right\} \min _{i}\left\{m_{i}\left(b^{*}(\lambda x)\right)\right\}}
$$

Therefore, when $\lambda \rightarrow+\infty$, one has $\frac{b^{*}(\lambda x)}{\min _{i}\left\{v_{i} / w_{i}\right\} \min _{i}\left\{m_{i}\left(b^{*}(\lambda x)\right)\right\}} \rightarrow+\infty$, and the convergence is uniform with respect to $x$ such that $w^{\top} x=1$. Consequently, $b^{*}(\lambda x) \rightarrow+\infty$ uniformly in $x$ such that $w^{\top} x=1$.

From this one deduces that $\lim _{\lambda \rightarrow+\infty} m_{i}\left(b^{*}(\lambda x)\right)<\lim _{\lambda \rightarrow+\infty} \mu_{i}(\lambda)$, because $m_{i}$ decreases, $\mu_{i}$ increases and Assumption 4 holds.

- Let us now show the second property on $b^{*}$. By definition one has, for any $x \in \mathbb{R}_{+}^{3} \backslash\{0\}$,

$$
b^{*}(x)=\sum_{i=1}^{3} v_{i} m_{i}\left(b^{*}(x)\right) x_{i} .
$$

Therefore, for any $\lambda, \lambda^{\prime} \geq 0$,

$$
\lambda \sum_{i=1}^{3} v_{i} m_{i}\left(b^{*}(\lambda x)\right) x_{i}-\lambda^{\prime} \sum_{i=1}^{3} v_{i} m_{i}\left(b^{*}\left(\lambda^{\prime} x\right)\right) x_{i}=b^{*}(\lambda x)-b^{*}\left(\lambda^{\prime} x\right)
$$

Subtracting and adding the term $\lambda \sum_{i=1}^{3} v_{i} m_{i}\left(b^{*}\left(\lambda^{\prime} x\right)\right) x_{i}$, one gets

$$
\lambda \sum_{i=1}^{3} v_{i}\left(m_{i}\left(b^{*}(\lambda x)\right)-m_{i}\left(b^{*}\left(\lambda^{\prime} x\right)\right)\right) x_{i}+\left(\lambda-\lambda^{\prime}\right) \sum_{i=1}^{3} v_{i} m_{i}\left(b^{*}\left(\lambda^{\prime} x\right)\right) x_{i}=b^{*}(\lambda x)-b^{*}\left(\lambda^{\prime} x\right)
$$

that is

$$
\left(\lambda-\lambda^{\prime}\right) \sum_{i=1}^{3} v_{i} m_{i}\left(b^{*}\left(\lambda^{\prime} x\right)\right) x_{i}=b^{*}(\lambda x)-b^{*}\left(\lambda^{\prime} x\right)-\lambda \sum_{i=1}^{3} v_{i}\left(m_{i}\left(b^{*}(\lambda x)\right)-m_{i}\left(b^{*}\left(\lambda^{\prime} x\right)\right)\right) x_{i}
$$

Assume e.g. $b^{*}(\lambda x)>b^{*}\left(\lambda^{\prime} x\right)$. Due to Assumption 1, the functions $m_{i}, i=1,2,3$, decrease. Therefore $m_{i}\left(b^{*}(\lambda x)\right)-m_{i}\left(b^{*}\left(\lambda^{\prime} x\right)\right)<0$, and we deduce from the previous identity that $\lambda>\lambda^{\prime}$.

One shows similarly that $b^{*}(\lambda x)<b^{*}\left(\lambda^{\prime} x\right)$ implies $\lambda<\lambda^{\prime}$. In conclusion, $\lambda<\lambda^{\prime} \Leftrightarrow b^{*}(\lambda x)<b^{*}\left(\lambda^{\prime} x\right)$, and this shows that $b^{*}$ is increasing.

\section{References}

[1] Hector Caraballo and Kevin King. Emergency department management of mosquito-borne illness: malaria, dengue, and West Nile virus. Emergency medicine practice, 16(5):1-23, 2014.

[2] Adnan I. Qureshi, editor. Zika virus disease: from origin to outbreak, chapter 2, Mosquito-Borne Diseases. Academic Press, 2018.

[3] World Health Organization et al. A global brief on vector-borne diseases. Technical report, World Health Organization, 2014.

[4] John T Roehrig and Robert S Lanciotti. Arboviruses. In Clinical Virology Manual, Fourth Edition, pages 387-407. American Society of Microbiology, 2009.

[5] Samir Bhatt, Peter W Gething, Oliver J Brady, Jane P Messina, Andrew W Farlow, Catherine L Moyes, John M Drake, John S Brownstein, Anne G Hoen, Osman Sankoh, et al. The global distribution and burden of dengue. Nature, 496(7446):504, 2013.

[6] Donald S Shepard, Eduardo A Undurraga, Yara A Halasa, and Jeffrey D Stanaway. The global economic burden of dengue: a systematic analysis. The Lancet infectious diseases, 16(8):935-941, 2016. 
[7] Smriti Sharma, Rubaljot Kooner, and Ramesh Arora. Insect pests and crop losses. In Breeding Insect Resistant Crops for Sustainable Agriculture, pages 45-66. Springer, 2017.

[8] E-C Oerke. Crop losses to pests. The Journal of Agricultural Science, 144(1):31-43, 2006.

[9] Angela E Douglas. Strategies for enhanced crop resistance to insect pests. Annual review of plant biology, 69:637-660, 2018.

[10] Catteruccia F. Shaw WR. Vector biology meets disease control: Using basic research to fight vectorborne diseases. Nature microbiology, 4:20-34, 2019.

[11] Janet Hemingway and Hilary Ranson. Insecticide resistance in insect vectors of human disease. Annual review of entomology, 45(1):371-391, 2000.

[12] Jacob C. Koella, Penelope A. Lynch, Matthew B. Thomas, and Andrew F. Read. Towards evolutionproof malaria control with insecticides. Evolutionary Applications, 2(4):469-480, 2009.

[13] J Vontas, E Kioulos, N Pavlidi, E Morou, A Della Torre, and Hilary Ranson. Insecticide resistance in the major dengue vectors Aedes albopictus and Aedes aegypti. Pesticide Biochemistry and Physiology, 104(2):126-131, 2012.

[14] Gabriela A Garcia, Ary A Hoffmann, Rafael Maciel-de Freitas, and Daniel AM Villela. Aedes aegypti insecticide resistance underlies the success (and failure) of Wolbachia population replacement. Scientific Reports, 10(1):1-9, 2020.

[15] Phillip J Daborn, Gaelle Le Goff, et al. The genetics and genomics of insecticide resistance. TRENDS in Genetics, 20(3):163-170, 2004.

[16] Pierrick Labbé, Haoues Alout, Luc Djogbénou, Nicole Pasteur, and Mylene Weill. Evolution of resistance to insecticide in disease vectors. In Genetics and evolution of infectious disease, pages 363-409. Elsevier, 2011.

[17] Josef Hofbauer and Karl Sigmund. Evolutionary games and population dynamics. Cambridge university press, 1998.

[18] Warren J Ewens. What Changes Has Mathematics Made to the Darwinian Theory? In The Mathematics of Darwin's Legacy, pages 7-26. Springer, 2011.

[19] Peter Schuster. Mathematical modeling of evolution. solved and open problems. Theory in Biosciences, 130(1):71-89, 2011.

[20] Peter Schuster. The mathematics of Darwin's theory of evolution: 1859 and 150 years later. In The Mathematics of Darwin's Legacy, pages 27-66. Springer, 2011.

[21] Reinhard Bürger. Some mathematical models in evolutionary genetics. In The Mathematics of Darwin's Legacy, pages 67-89. Springer, 2011.

[22] Thierry Huillet and Servet Martinez. Discrete evolutionary genetics. Multiplicative fitnesses and the mutation-fitness balance. Applied Mathematics, Vol 2(no 1):pp. 11-22, January 2011.

[23] Peter Schuster and Karl Sigmund. Replicator dynamics. Journal of theoretical biology, 100(3):533$538,1983$.

[24] Josef Hofbauer, Peter Schuster, and Karl Sigmund. Game dynamics in Mendelian populations. Biological Cybernetics, 43(1):51-57, 1982.

[25] Susana Barbosa and Ian M Hastings. The importance of modelling the spread of insecticide resistance in a heterogeneous environment: the example of adding synergists to bed nets. Malaria journal, 11(1):258, 2012.

[26] Bethany Levick, Andy South, and Ian M Hastings. A two-locus model of the evolution of insecticide resistance to inform and optimise public health insecticide deployment strategies. PLoS computational biology, 13(1):e1005327, 2017. 
[27] C Robert Taylor and JC Headley. Insecticide resistance and the evaluation of control strategies for an insect population. The Canadian Entomologist, 107(3):237-242, 1975.

[28] Hugh N Comins. The development of insecticide resistance in the presence of migration. Journal of theoretical biology, 64(1):177-197, 1977.

[29] Charles E Taylor and George P Georghiou. Suppression of insecticide resistance by alteration of gene dominance and migration. Journal of Economic Entomology, 72(1):105-109, 1979.

[30] James F Crow, Motoo Kimura, et al. An introduction to population genetics theory. An introduction to population genetics theory., 1970.

[31] CF Curtis. Theoretical models of the use of insecticide mixtures for the management of resistance. Bulletin of entomological research, 75(2):259-266, 1985.

[32] GS Mani. Evolution of resistance in the presence of two insecticides. Genetics, 109(4):761-783, 1985.

[33] Andy South and Ian M Hastings. Insecticide resistance evolution with mixtures and sequences: a model-based explanation. Malaria journal, 17(1):80, 2018.

[34] Andrew F Read, Penelope A Lynch, and Matthew B Thomas. How to make evolution-proof insecticides for malaria control. PLoS biology, 7(4):e1000058, 2009.

[35] Stephen A Gourley, Rongsong Liu, and Jianhong Wu. Slowing the evolution of insecticide resistance in mosquitoes: a mathematical model. Proceedings of the Royal Society A: Mathematical, Physical and Engineering Sciences, 467(2132):2127-2148, 2011.

[36] Dirk Langemann, Otto Richter, and Antje Vollrath. Multi-gene-loci inheritance in resistance modeling. Mathematical biosciences, 242(1):17-24, 2013.

[37] Helio Schechtman and Max O Souza. Costly inheritance and the persistence of insecticide resistance in Aedes aegypti populations. PloS one, 10(5):e0123961, 2015.

[38] Yazhi Li and Xianning Liu. Modeling and control of mosquito-borne diseases with Wolbachia and insecticides. Theoretical Population Biology, 2020.

[39] Wlodzimierz Klonowski. Simplifying principles for chemical and enzyme reaction kinetics. Biophysical Chemistry, 18(2):73-87, 1983.

[40] Joseph Felsenstein. Theoretical evolutionary genetics. University of Washington, Seattle, 2005.

[41] A.W.F. Edwards. Punnett's square. Studies in History and Philosophy of Science Part C: Studies in History and Philosophy of Biological and Biomedical Sciences, 43(1):219 - 224, 2012. Data-Driven Research in the Biological and Biomedical Sciences On Nature and Normativity: Normativity, Teleology, and Mechanism in Biological Explanation.

[42] EA Elbadry and MSF Tawfik. Life cycle of the mite Adactylidium sp. (Acarina: Pyemotidae), a predator of thrips eggs in the United Arab Republic. Annals of the Entomological Society of America, 59(3):458-461, 1966.

[43] Stephen Jay Gould. The panda's thumb: More reflections in natural history. WW Norton \& company, 2010.

[44] Craig H Welch. Shortest reproductive life. University of Florida Book of Insect Records, 1998.

[45] Robert E O'Malley Jr. Singular perturbation methods for ordinary differential equations, volume 89. Springer Science \& Business Media, 1991.

[46] AN Tikhonov, Vasil'eva AB, and Sveshnikov AG. Differential Equations. Springer, New York, 1980.

[47] Jack Carr. Applications of centre manifold theory, volume 35. Springer Science \& Business Media, 2012. 
[48] Laurent Duret. Neutral theory: the null hypothesis of molecular evolution. Nature Education, 2008.

[49] Edward O Wilson and William H Bossert. A primer of population biology. Sinauer Associates Sunderland, MA, 1971.

[50] Scott Freeman and Jon C Herron. Evolutionary analysis. Number QH 366.2. F73 2007. Pearson Prentice Hall Upper Saddle River, NJ, 2007.

[51] I. Barbălat. Systèmes d'équations différentielles d'oscillations non linéaires. Rev. Math. Pures Appl., $4: 267-270,1959$.

[52] Bálint Farkas and Sven-Ake Wegner. Variations on Barbălat's lemma. The American Mathematical Monthly, 123(8):825-830, 2016.

[53] Thomas M Priester and George P Georghiou. Induction of high resistance to permethrin in Culex pipiens quinquefasciatus. Journal of economic entomology, 71(2):197-200, 1978.

[54] CF Curtis, LM Cook, and RJ Wood. Selection for and against insecticide resistance and possible methods of inhibiting the evolution of resistance in mosquitoes. Ecological Entomology, 3(4):273-287, 1978.

[55] D Bourguet, A Genissel, and M Raymond. Insecticide resistance and dominance levels. Journal of economic entomology, 93(6):1588-1595, 2000.

[56] Peter S Dawson. Linkage and the elimination of deleterious mutant genes from experimental populations. Genetica, 41(1):147-169, 1970.

[57] JC Helps, ND Paveley, and F van den Bosch. Identifying circumstances under which high insecticide dose increases or decreases resistance selection. Journal of theoretical biology, 428:153-167, 2017.

[58] Adi Kliot and Murad Ghanim. Fitness costs associated with insecticide resistance. Pest management science, 68(11):1431-1437, 2012.

[59] Ary A Hoffmann and Michael Turelli. Facilitating Wolbachia introductions into mosquito populations through insecticide-resistance selection. Proceedings of the Royal Society B: Biological Sciences, 280(1760):20130371, 2013.

[60] Paul Keddy. Plants and vegetation: origins, processes, consequences. Cambridge University Press, 2007.

[61] Luke Alphey. Genetic control of mosquitoes. Annual review of entomology, 59, 2014.

[62] Ary A Hoffmann, Perran A Ross, and Gordana Rašić. Wolbachia strains for disease control: ecological and evolutionary considerations. Evolutionary applications, 8(8):751-768, 2015. 